\title{
Morpholine-Based Immonium and Halogenoamidinium Salts as Coupling Reagents in Peptide Synthesis
}

\author{
Ayman El-Faham, ${ }^{\mathrm{a}, \mathrm{b},{ }^{*}}$ Fernando Albericio, ${ }^{\mathrm{a}, \mathrm{c}, \mathrm{d}^{*}}$ \\ ${ }^{a}$ Institute for Research in Biomedicine, Barcelona Science Park, Josep Samitier 1, \\ 08028-Barcelona, Spain \\ ${ }^{b}$ Department of Chemistry, Faculty of Science, Alexandria University, Ibrahimia 21321, \\ Alexandria, Egypt \\ ${ }^{c}$ Department of Organic Chemistry, University of Barcelona, Martí i Franqués 1-11, \\ 08028-Barcelona, Spain \\ ${ }^{d}$ CIBER-BBN, Networking Centre on Bioengineering, Biomaterials and Nanomedicine, \\ Barcelona Science Park, Josep Samitier 1-5, E-08028 Barcelona, Spain
}

\section{Supporting Information}

Content:

General Procedures ........Pag 2

Copies of ${ }^{1} \mathrm{H}$ - and ${ }^{13} \mathrm{C}-\mathrm{NMR}$ Spectra of Compounds 3ii, 4i, 5c, 5d, 5e, 5f, 5g, 6a, $6 \mathrm{~b}$, and $7 \ldots . . . . .$. Pag $3-22$

X-ray Tables .............Pag 23-36 


\section{General Procedures}

TLC was performed on silica plates $(8 \times 4 \mathrm{~cm})$ using suitable solvent systems and visualization by a Spectroline UV Lamp Model CM-10 (254 nm). Melting points were obtained in open capillary tubes and were uncorrected. Infrared spectra (IR) were recorded on a FT-IR instrument as $\mathrm{KBr}$ pellets. The absorption bands $\left(v_{\max }\right)$ were given in

wave numbers $\left(\mathrm{cm}^{-1}\right)$. NMR spectra were recorded on a $400 \mathrm{MHz}$ spectrometer at room temperature. Tetramethylsilane (TMS) was used as reference for all NMR spectra with chemical shifts reported as ppm relative to TMS. HPLC analyses were carried out using

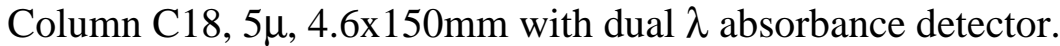

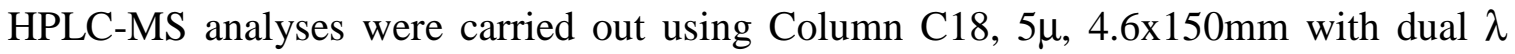
absorbance detector. All solvents used for recrystallization, extraction, column chromatography and TLC were of commercial grade, distilled before use and stored under dry conditions. 


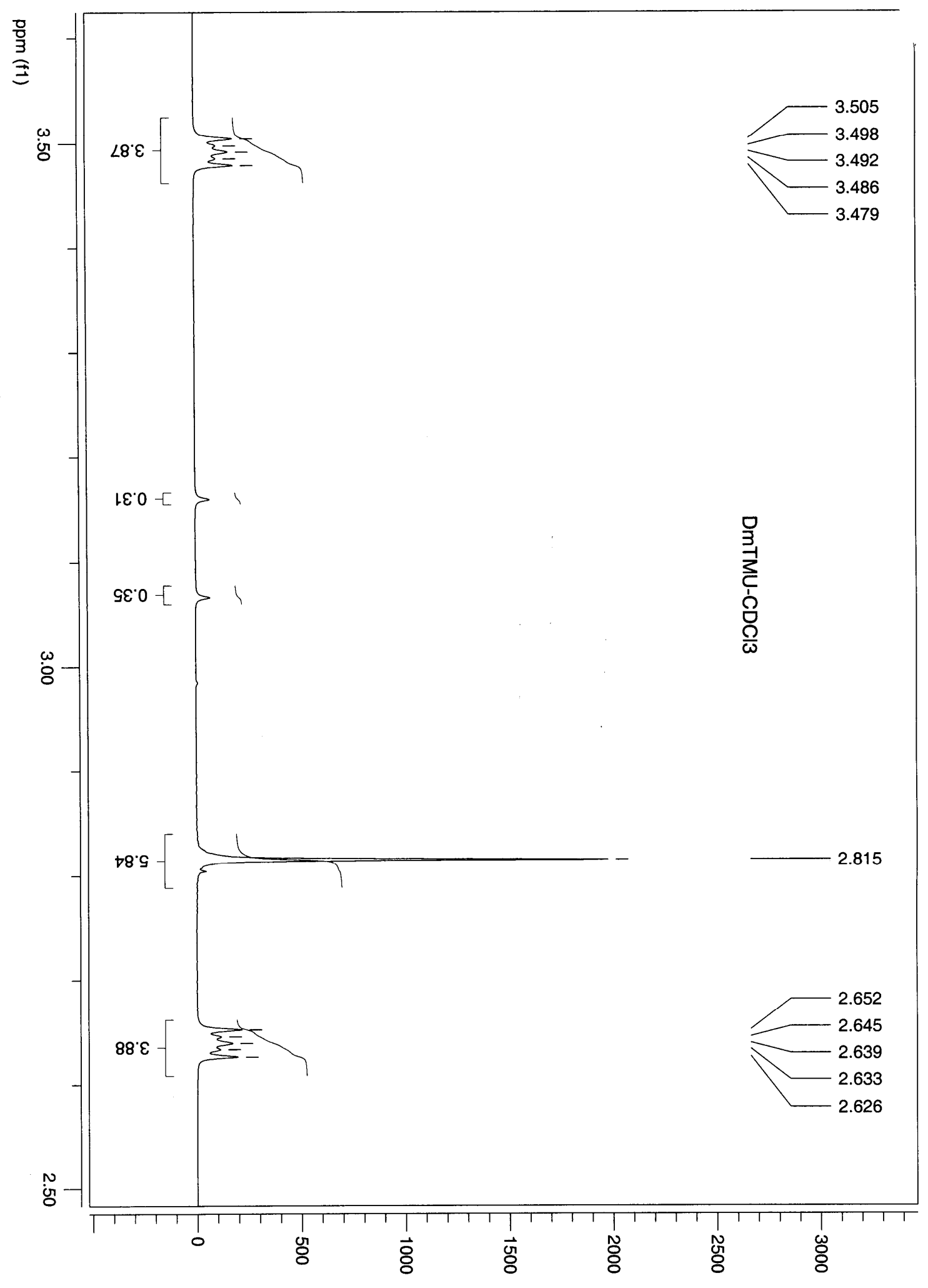

3 ii, H-NMR 


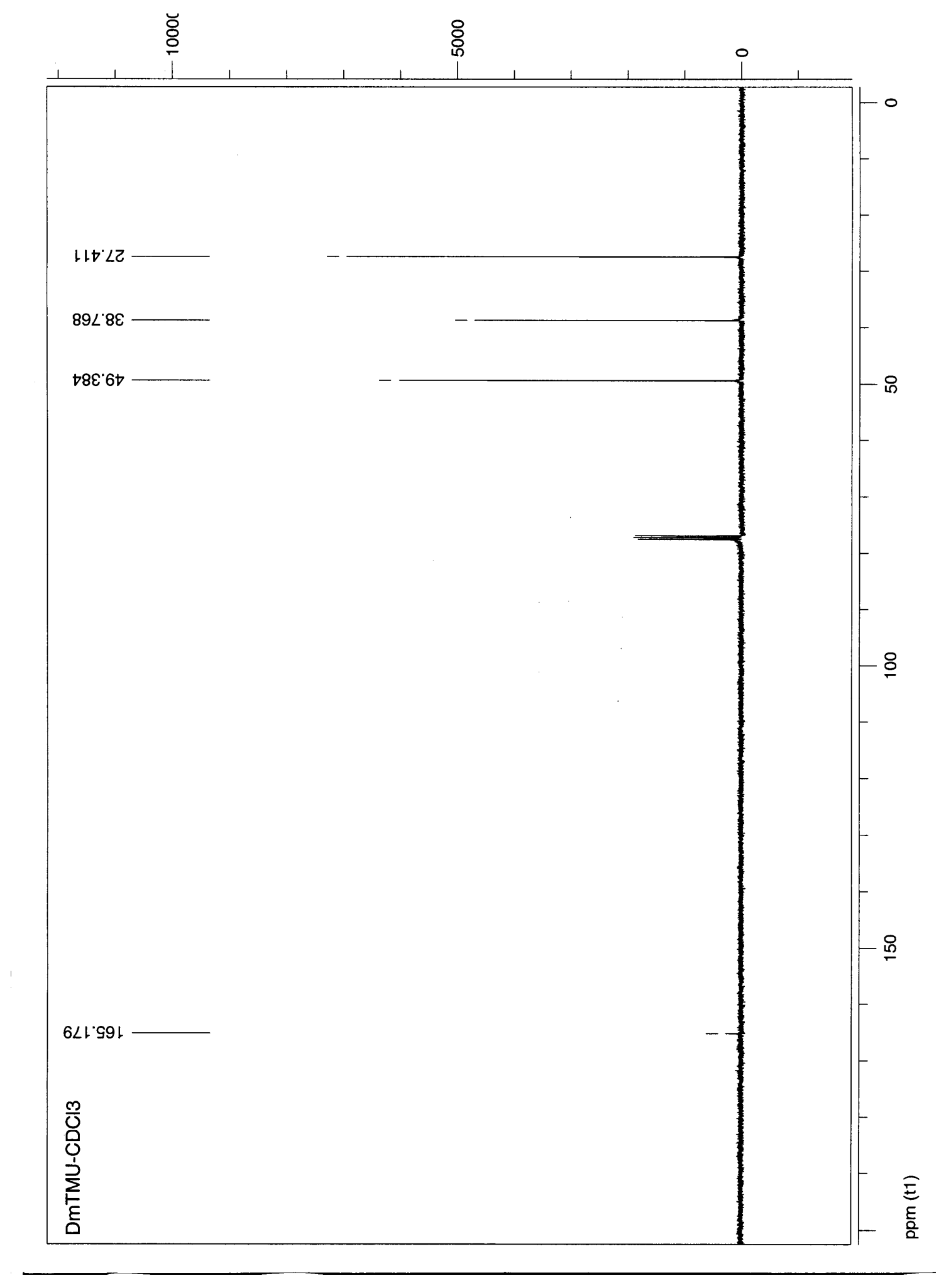

3ii, C-NMR 


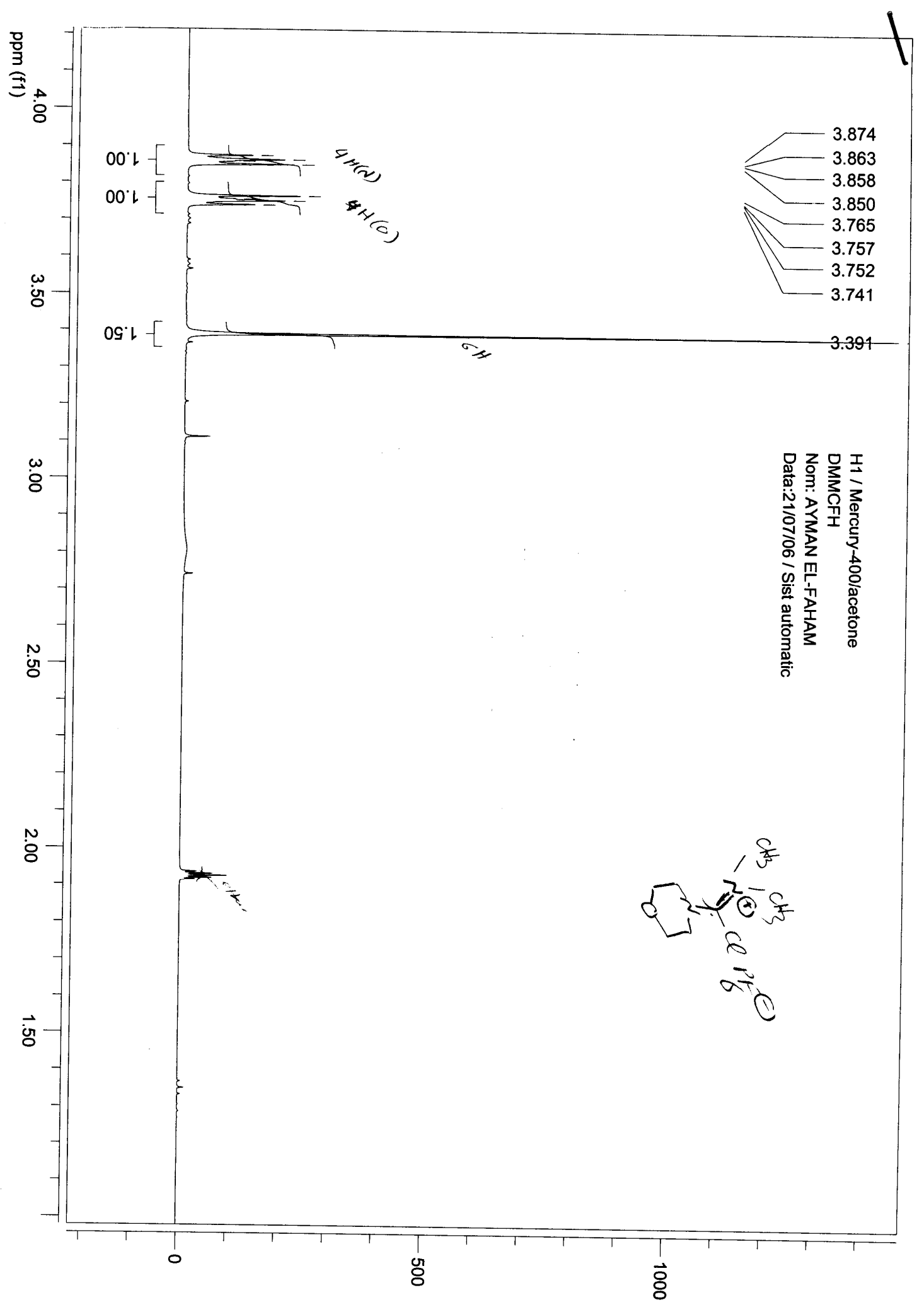

\section{4i, H-NMR}




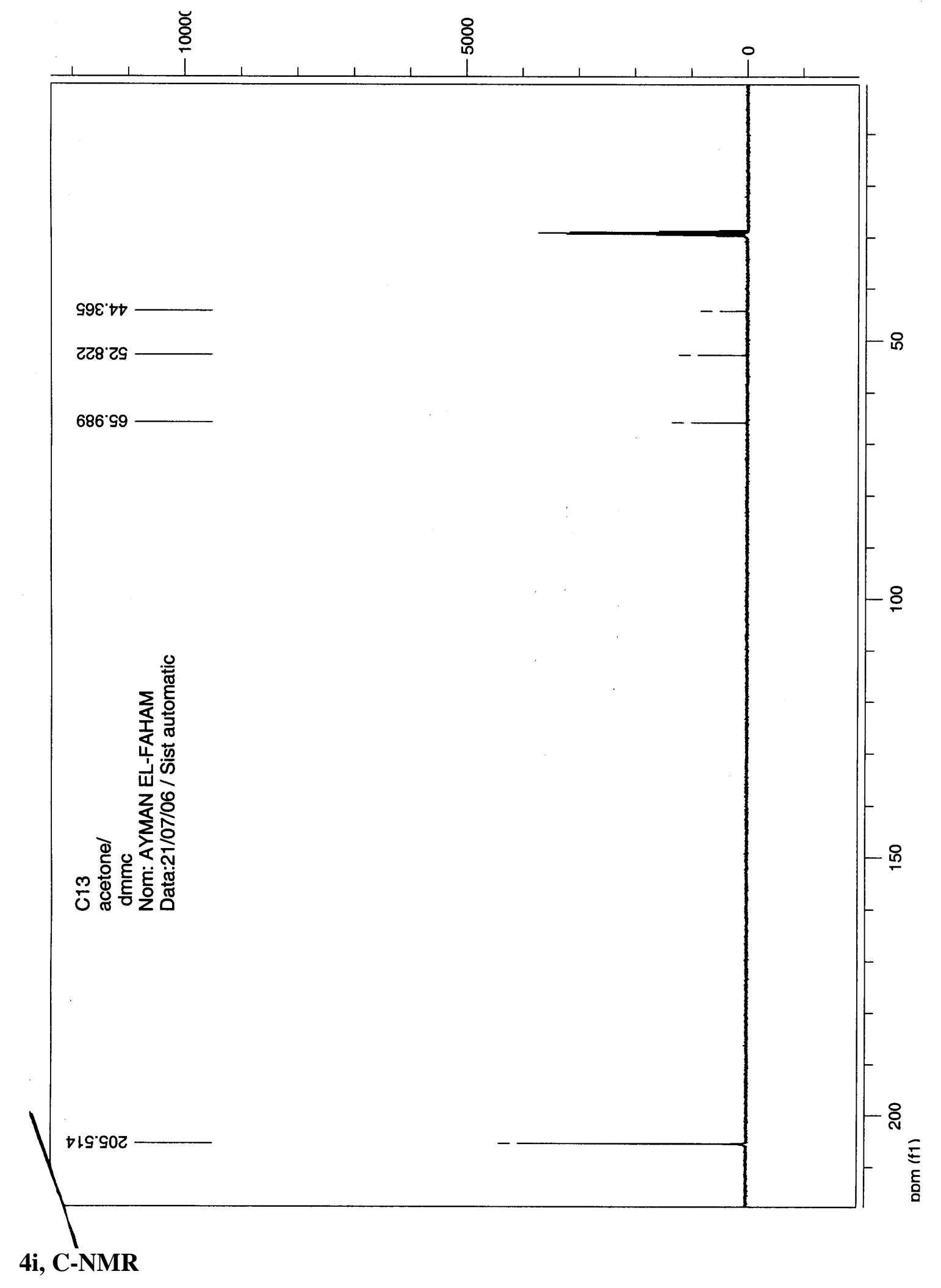




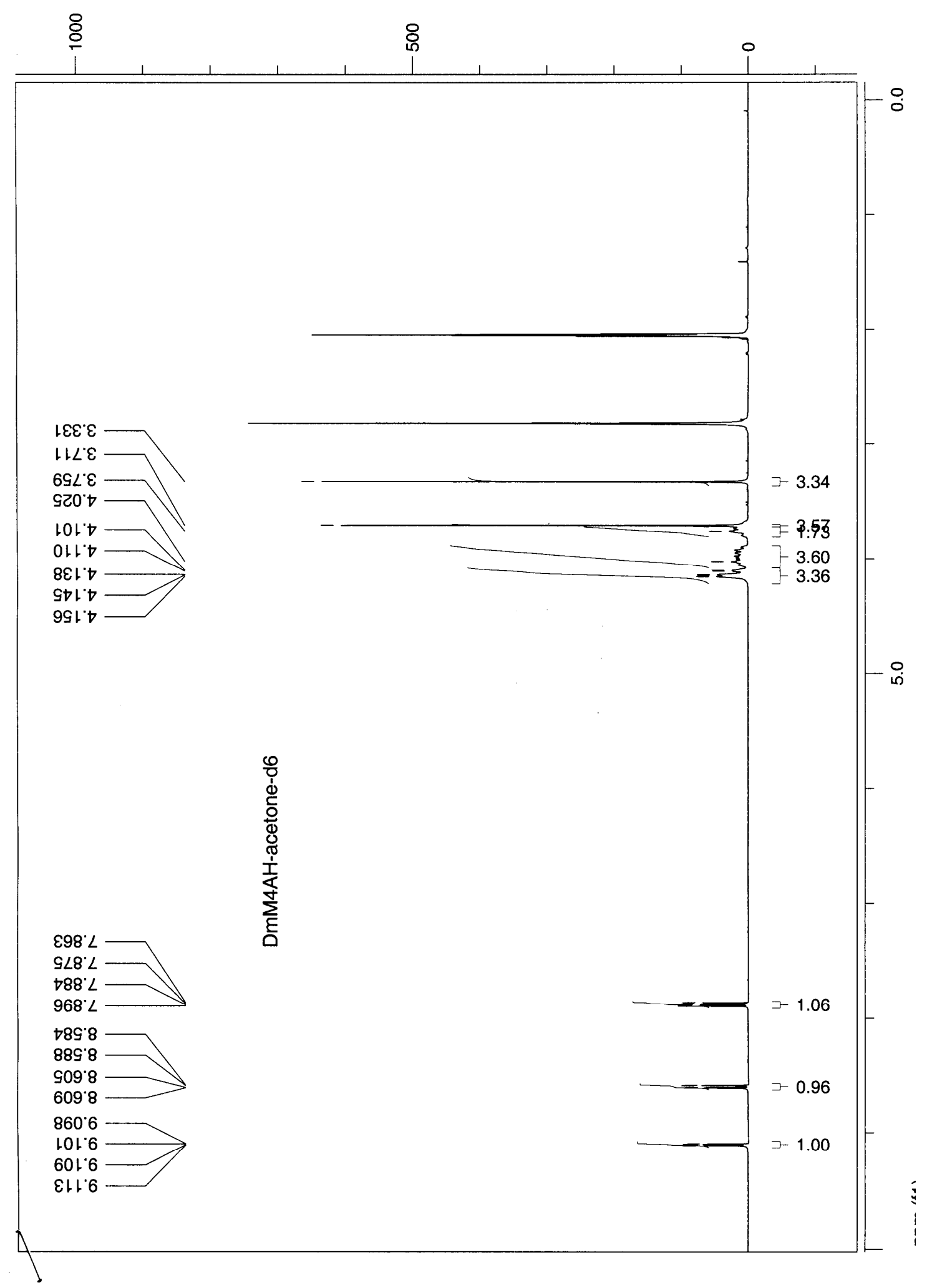

5c, H-NMR 


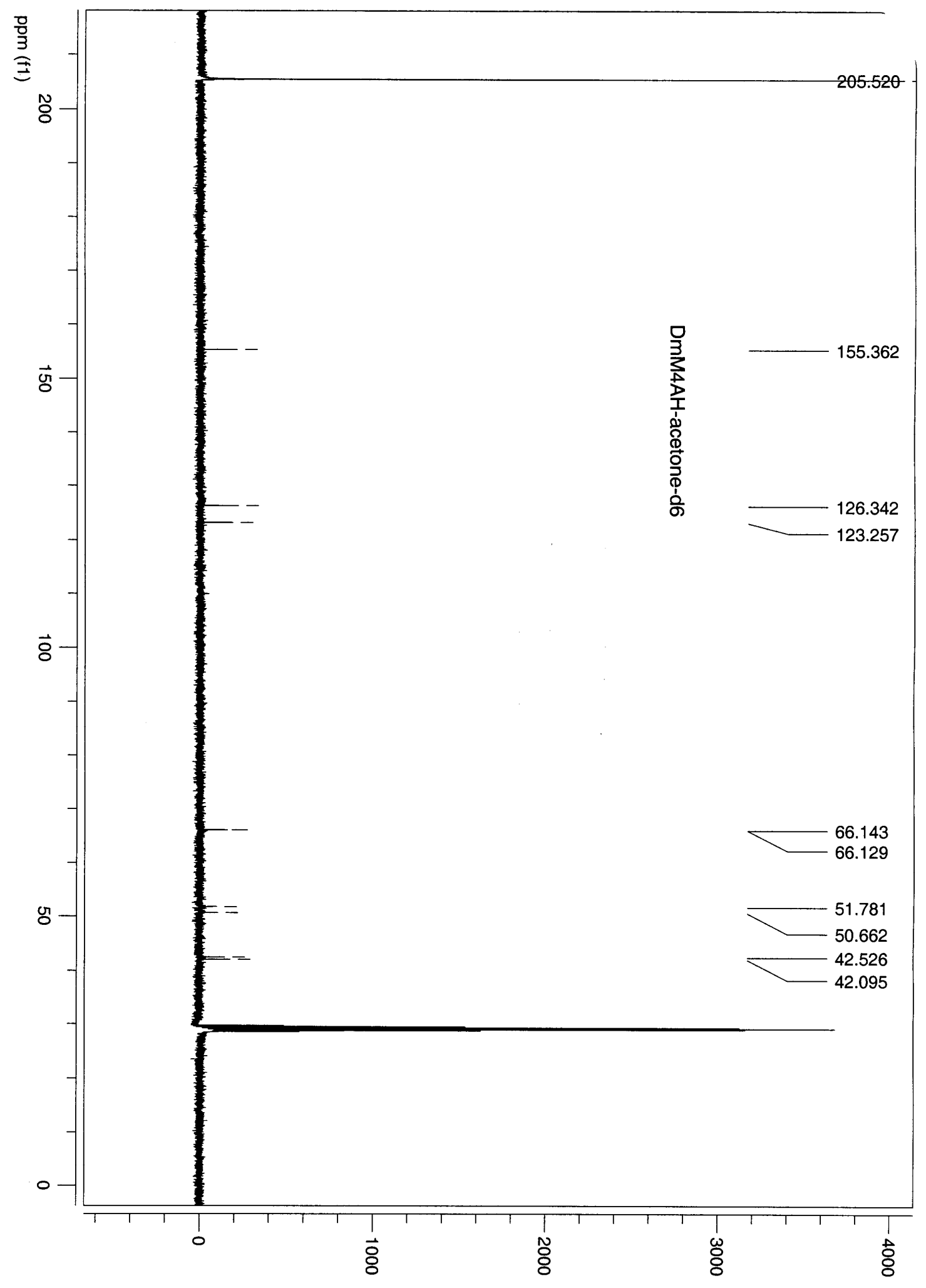

5c, C-NMR 


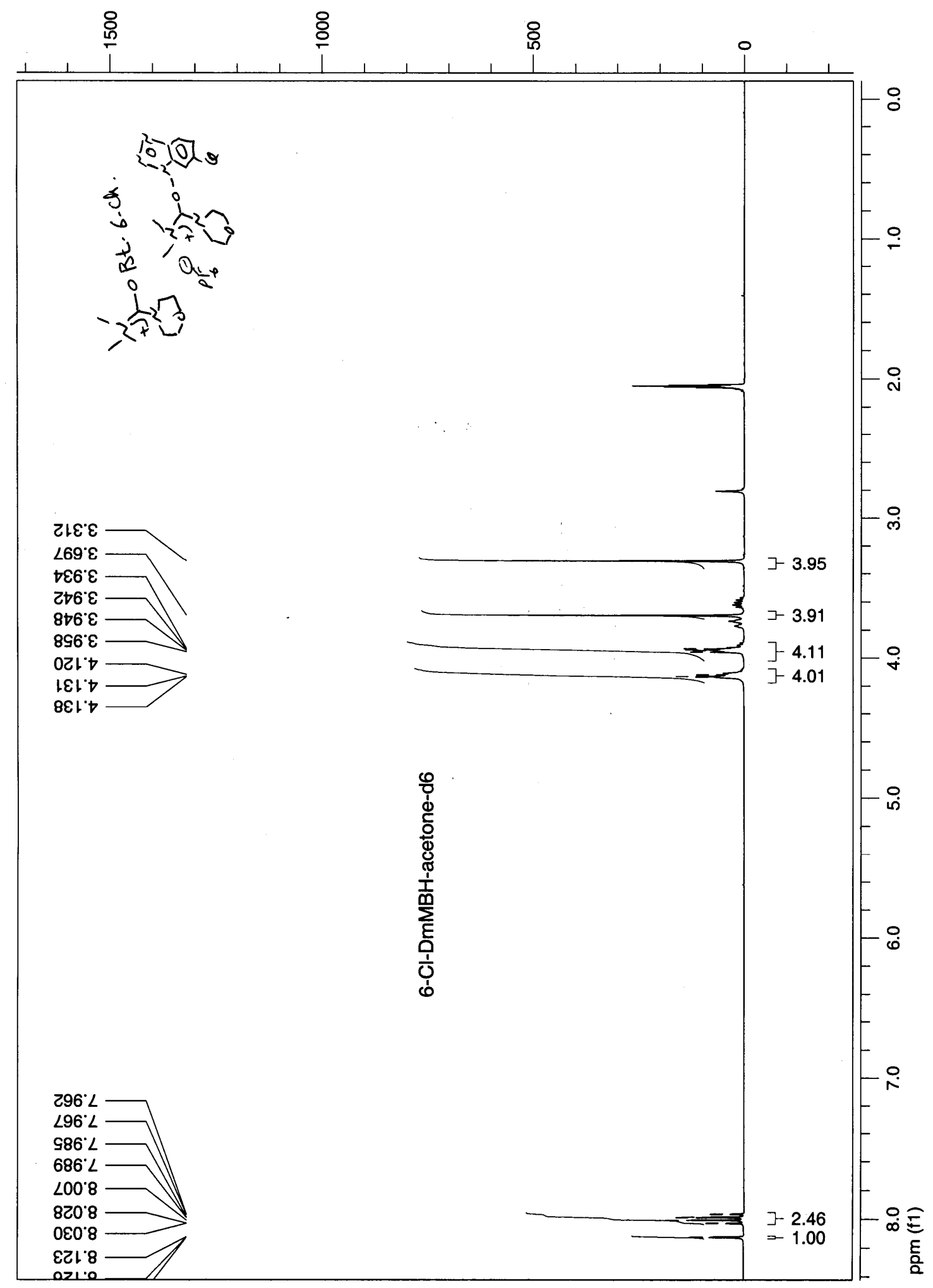

5d. H-NMR 


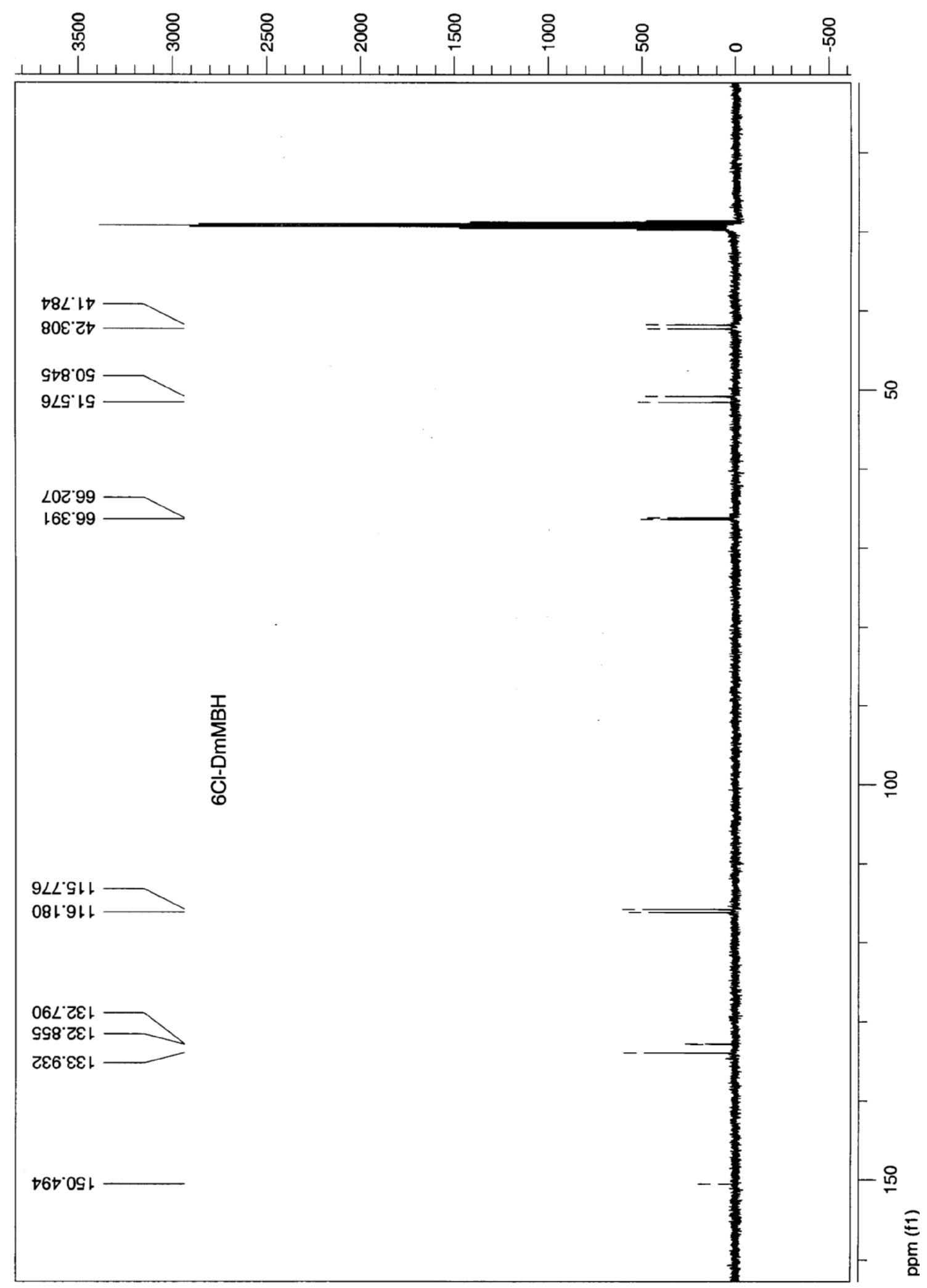

5d, C-NMR 


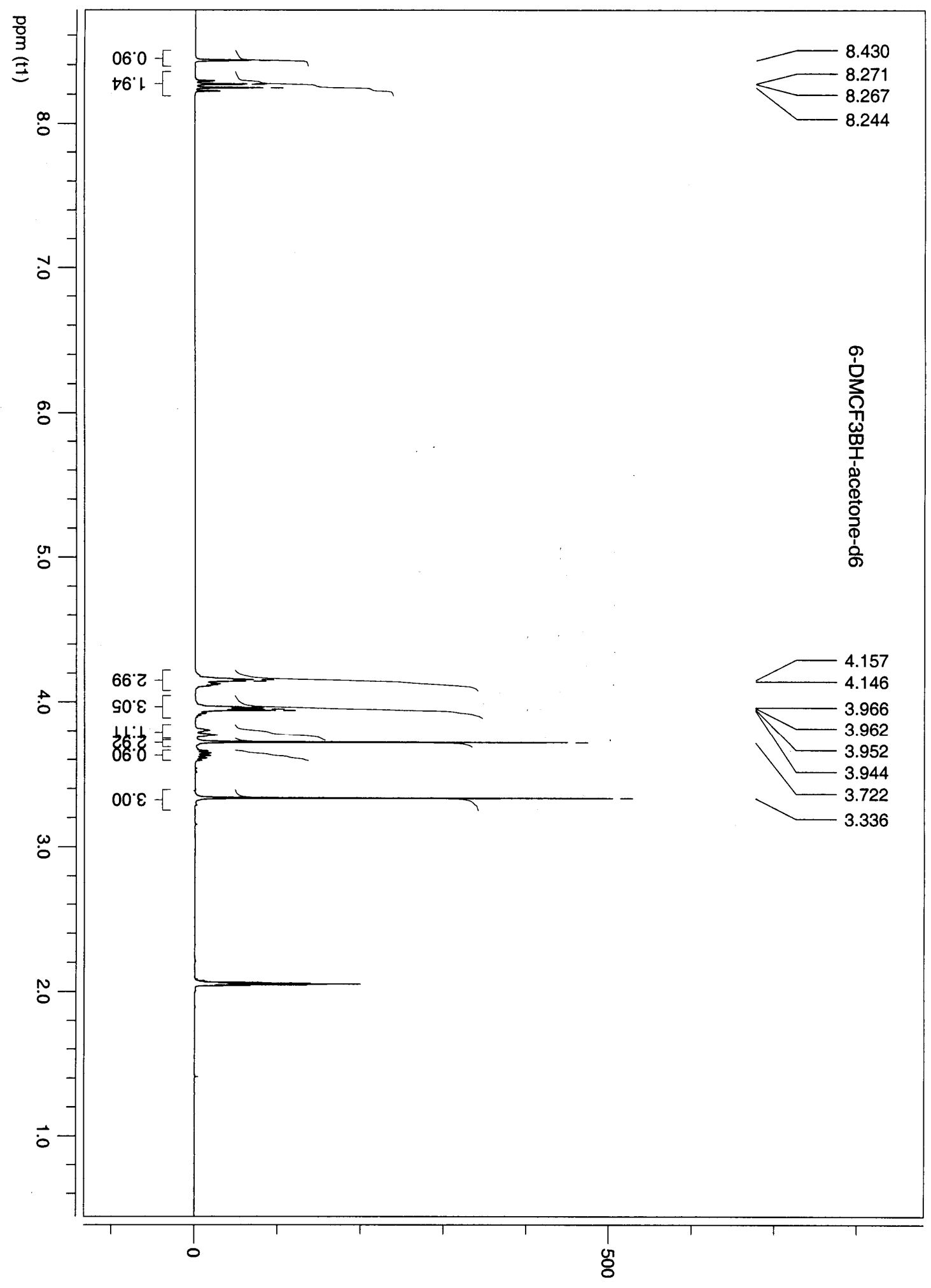

5e, H-NMR 


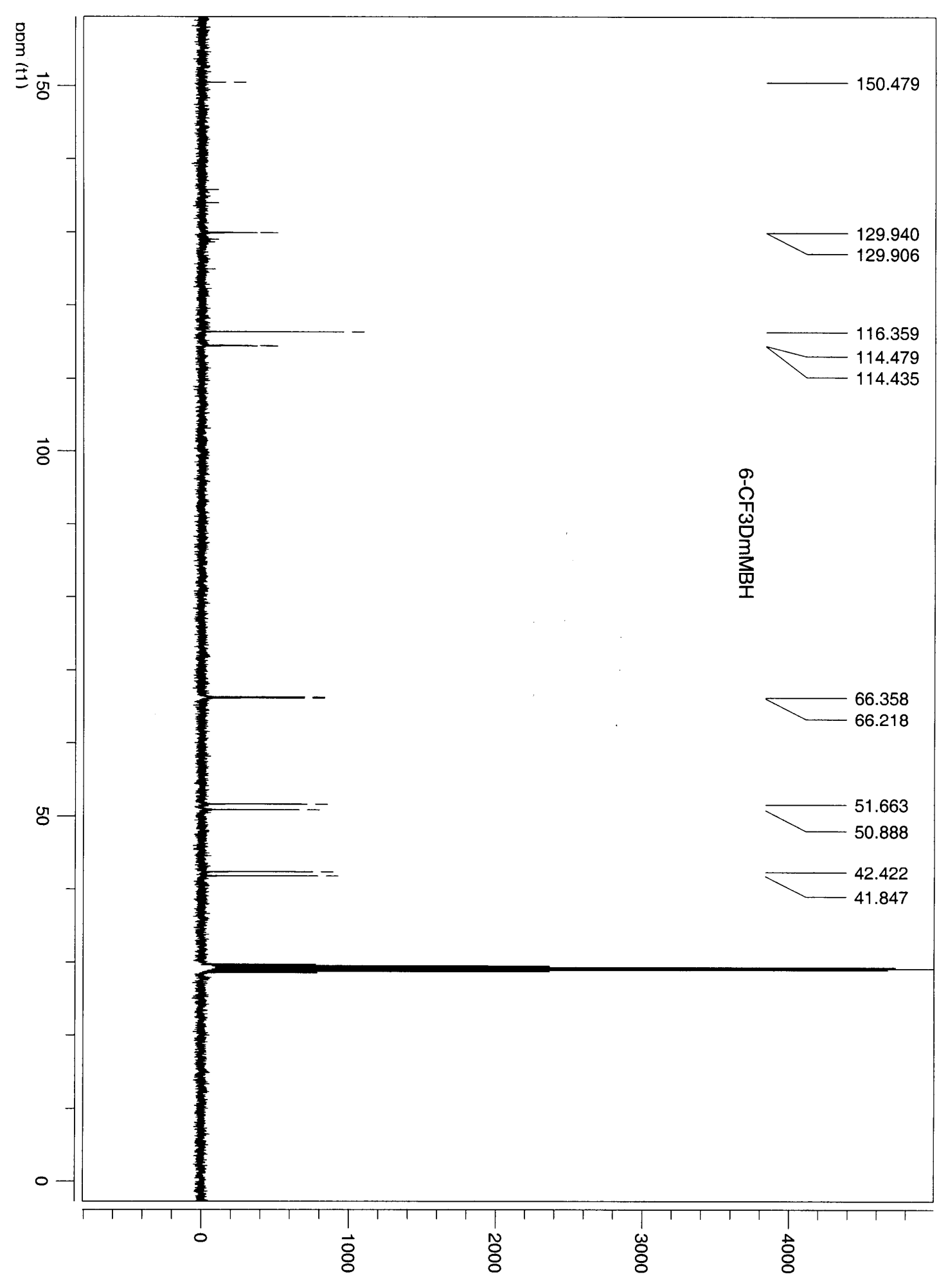

5e, C-NMR 


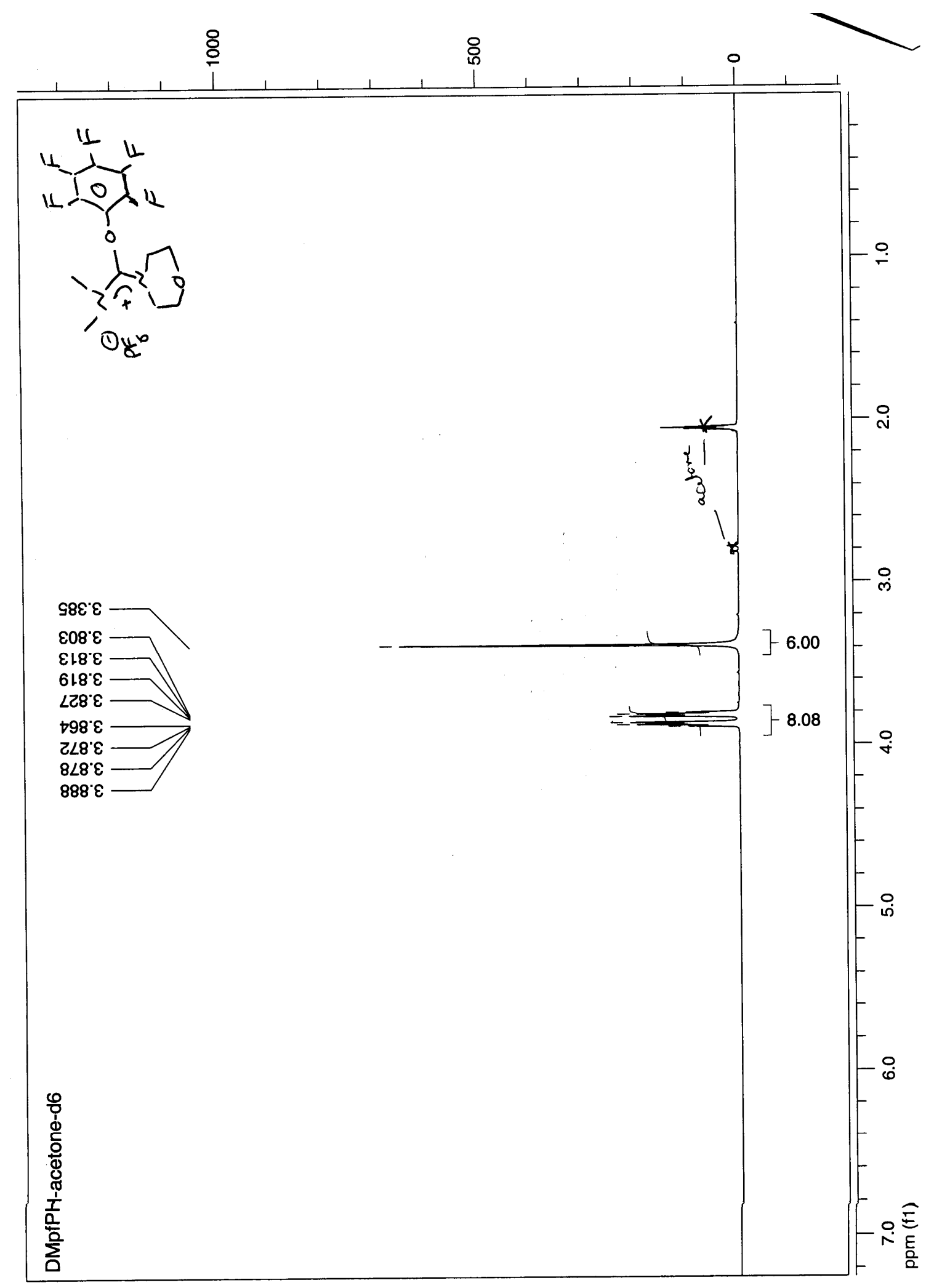

5f, H-NMR 


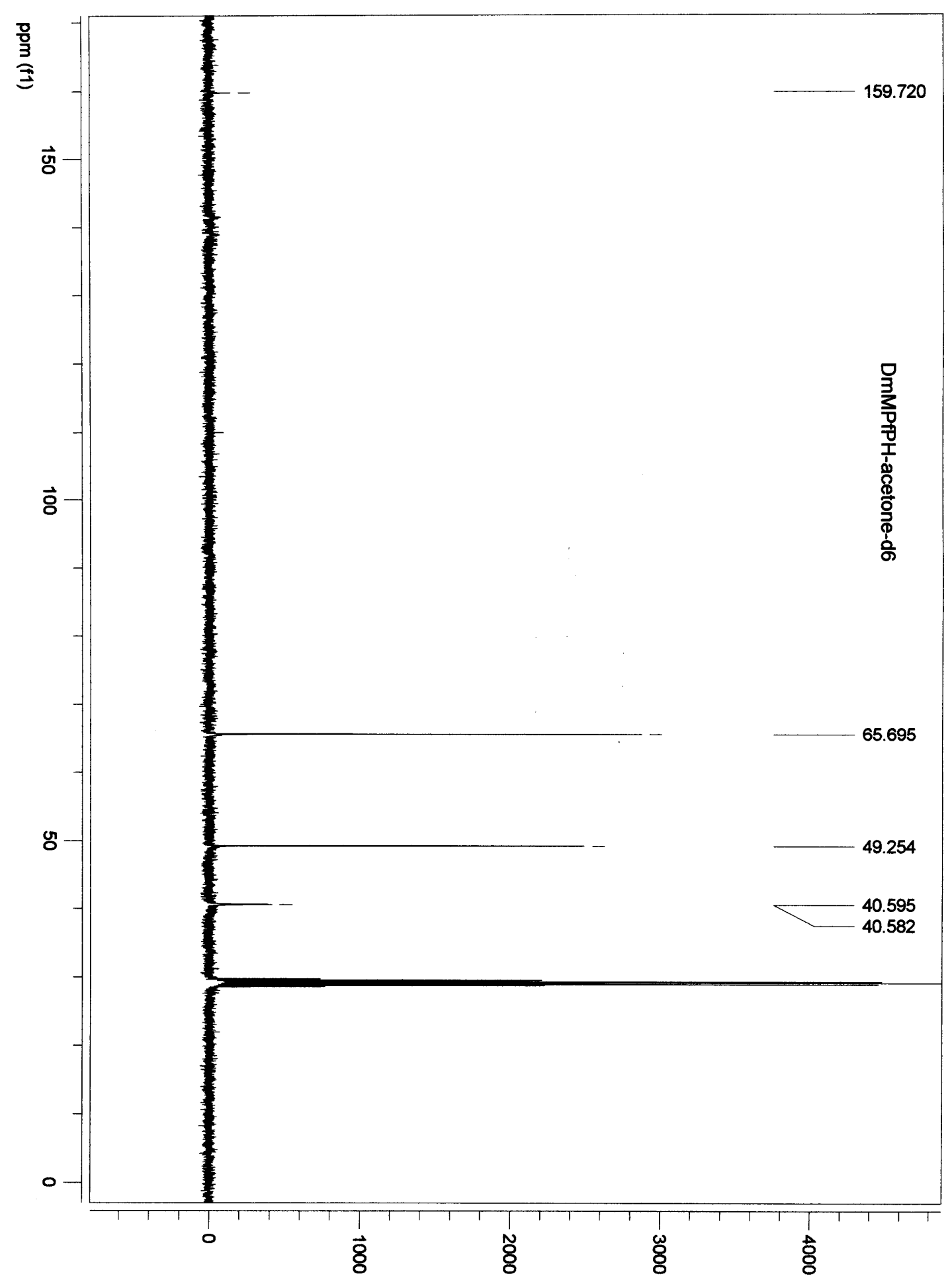

5f, C-NMR 


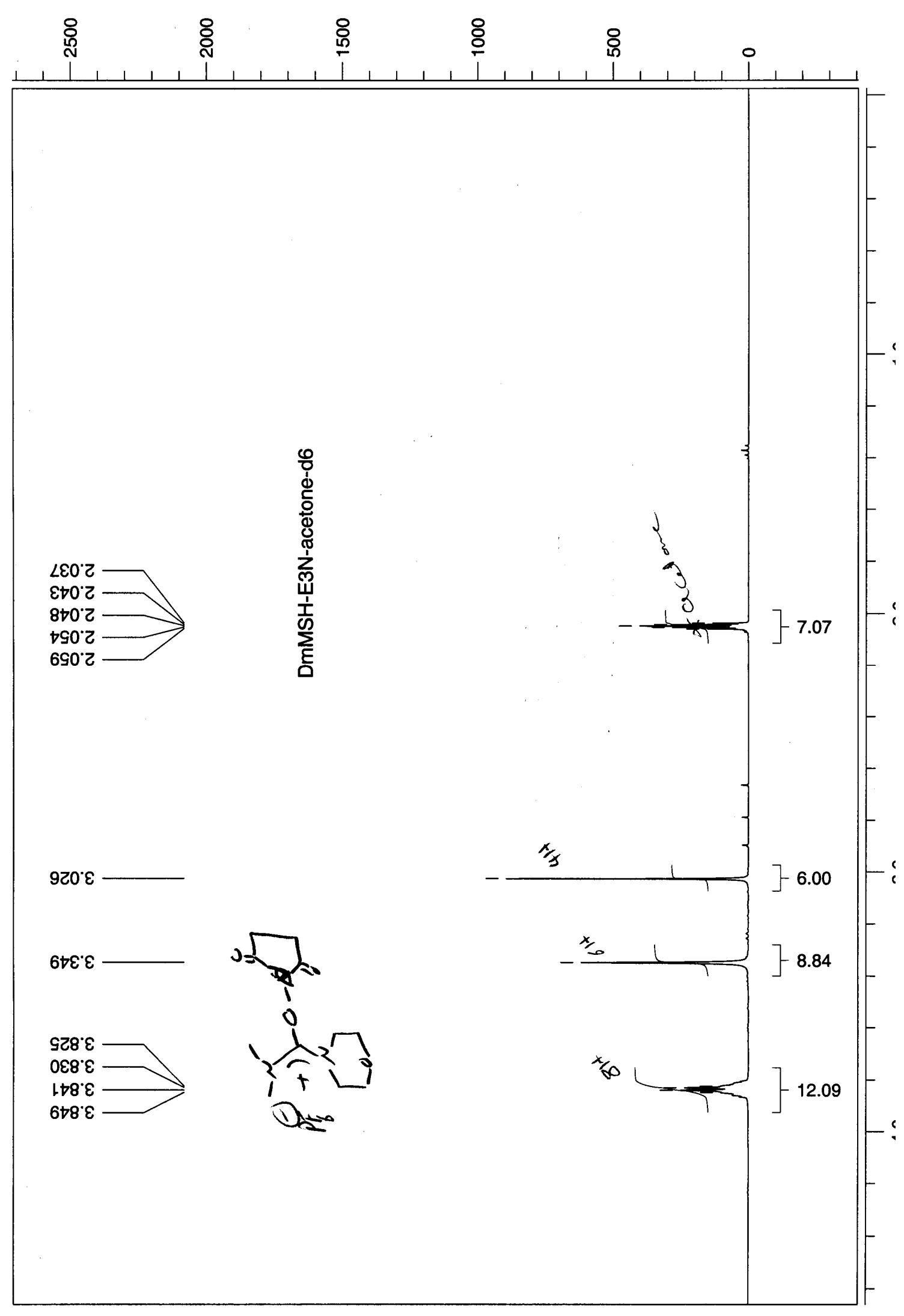




\section{5g, H-NMR}

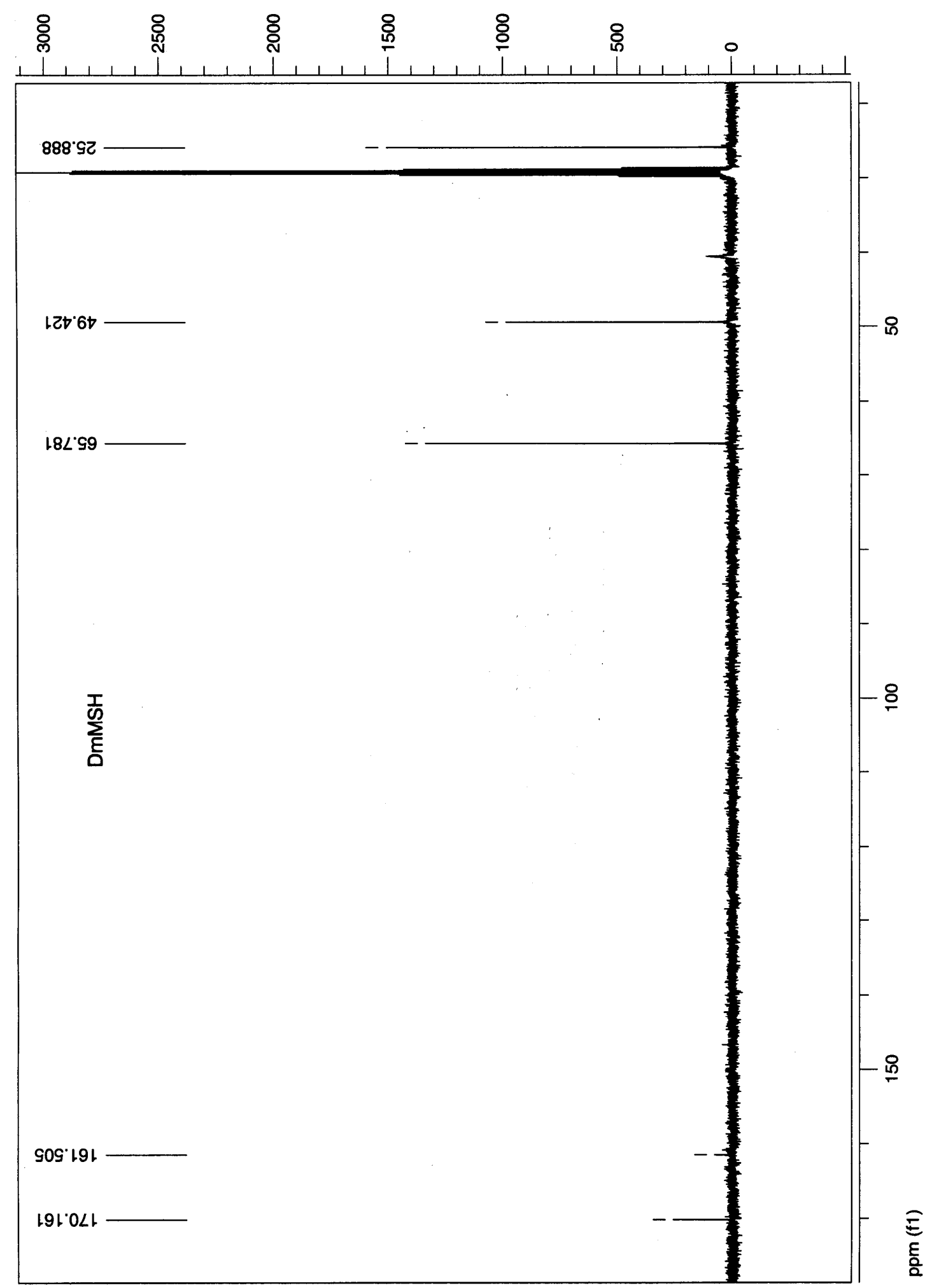

5g, C-NMR 


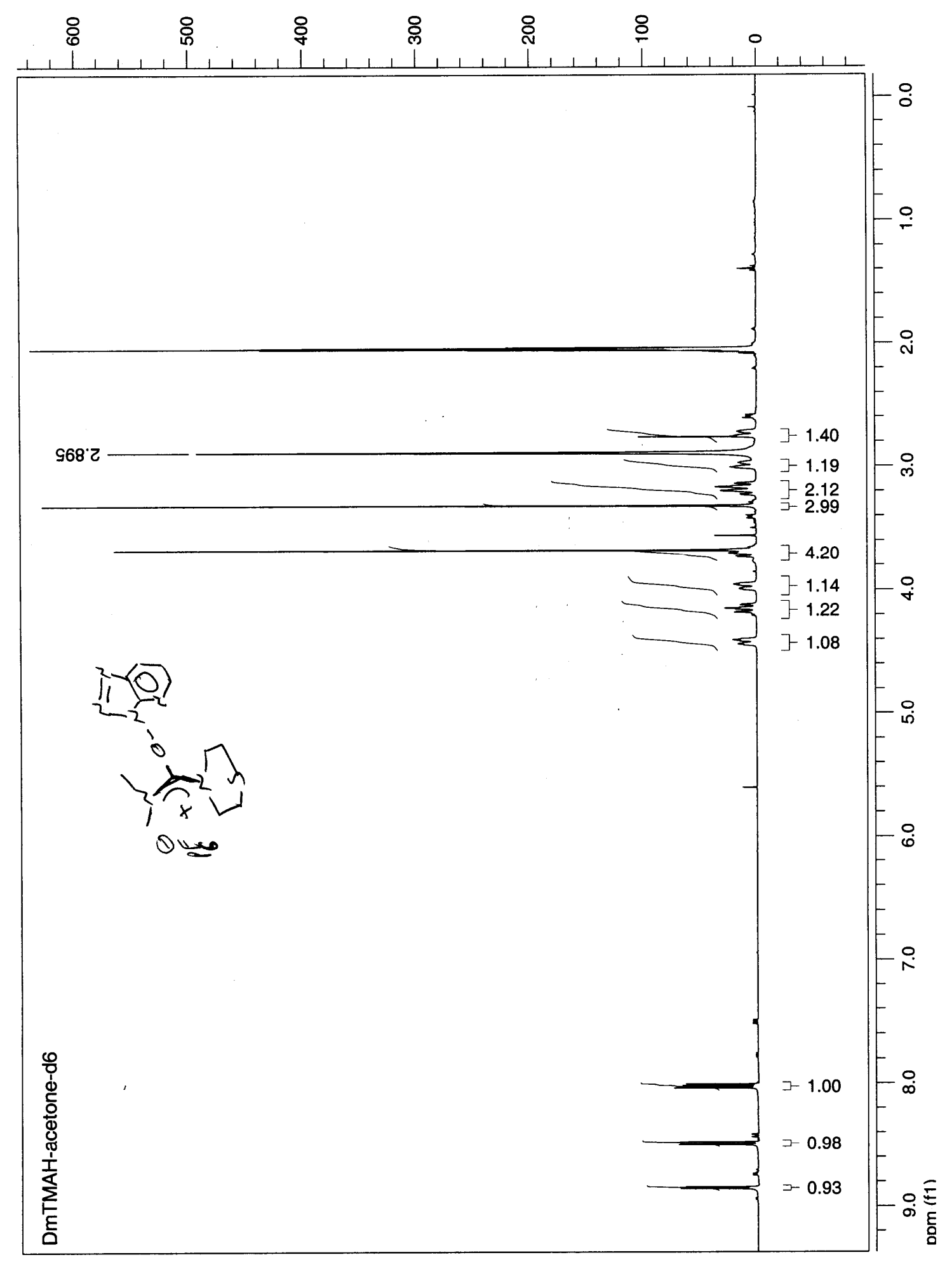

6a, H-NMR 


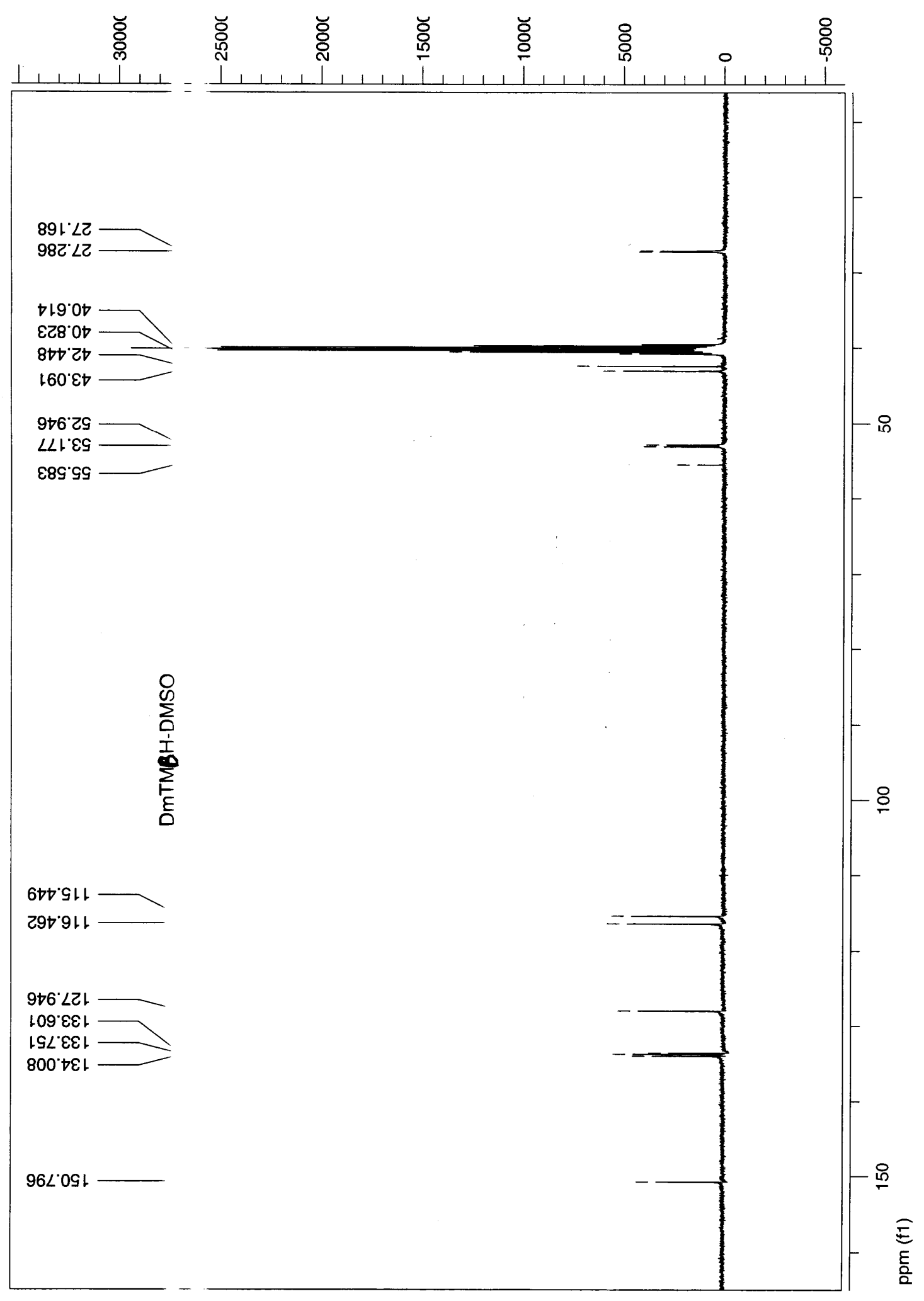

6a, C-NMr 


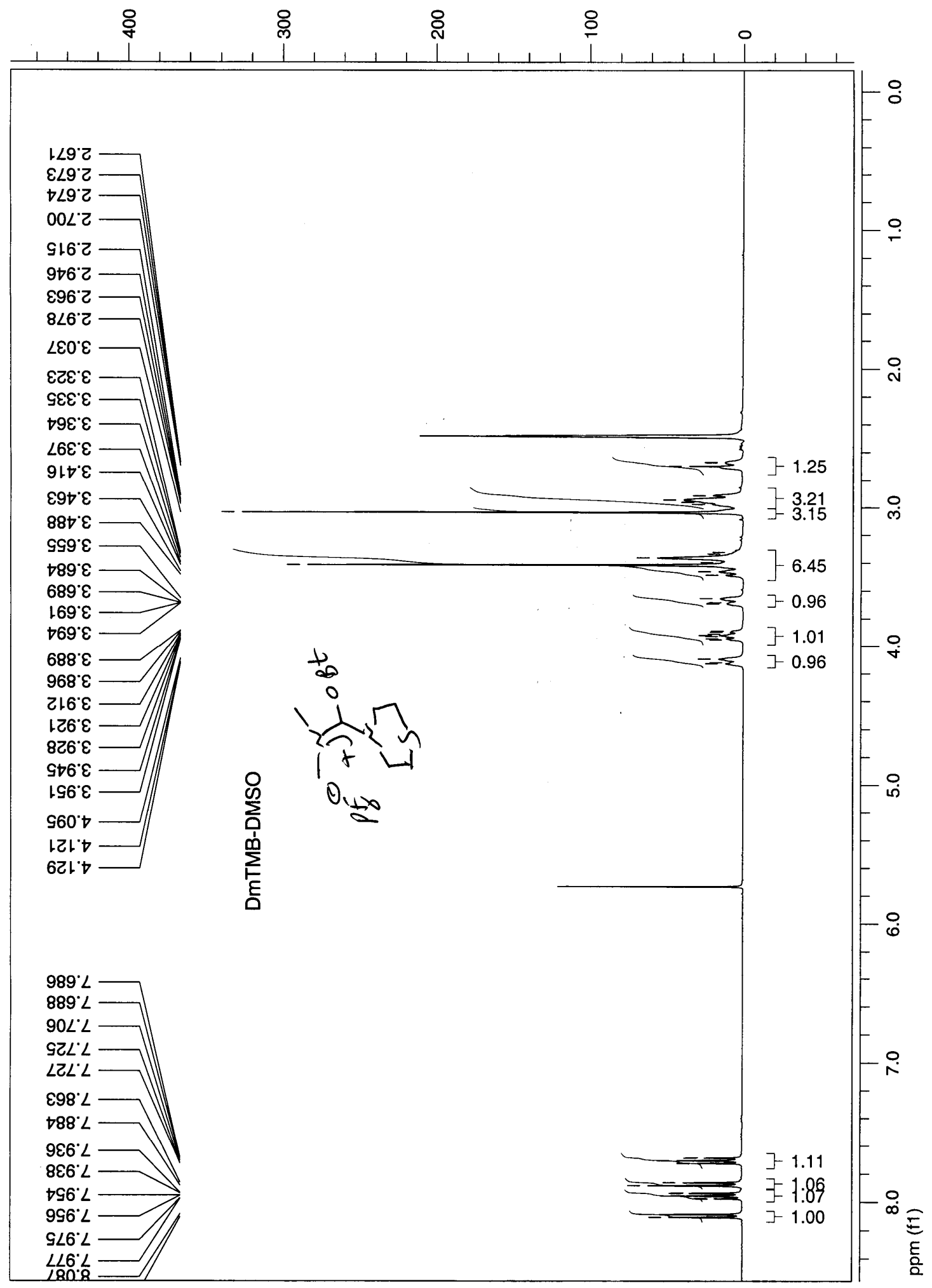

\section{6b, H-NMR}




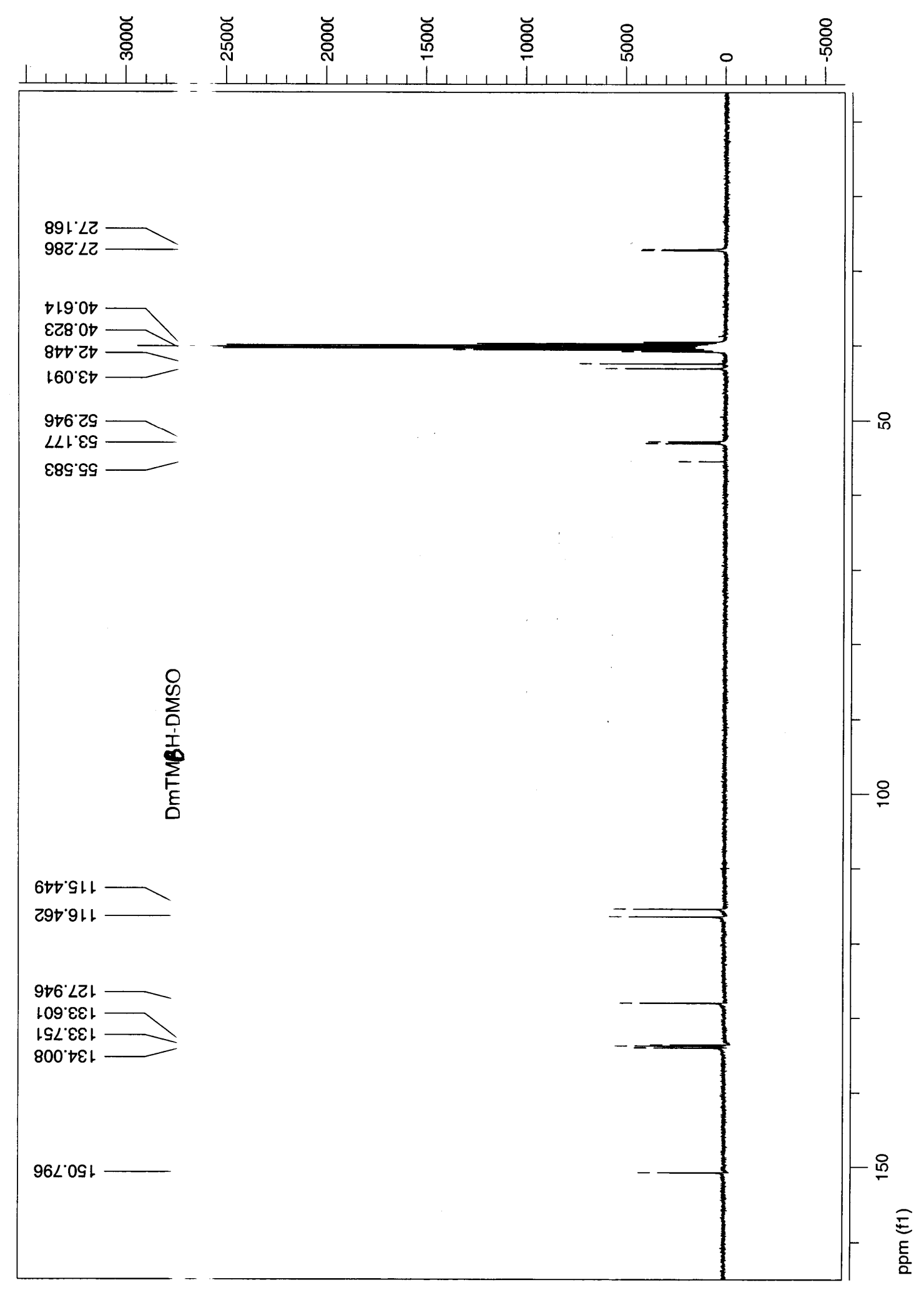

6b, C-NMR 


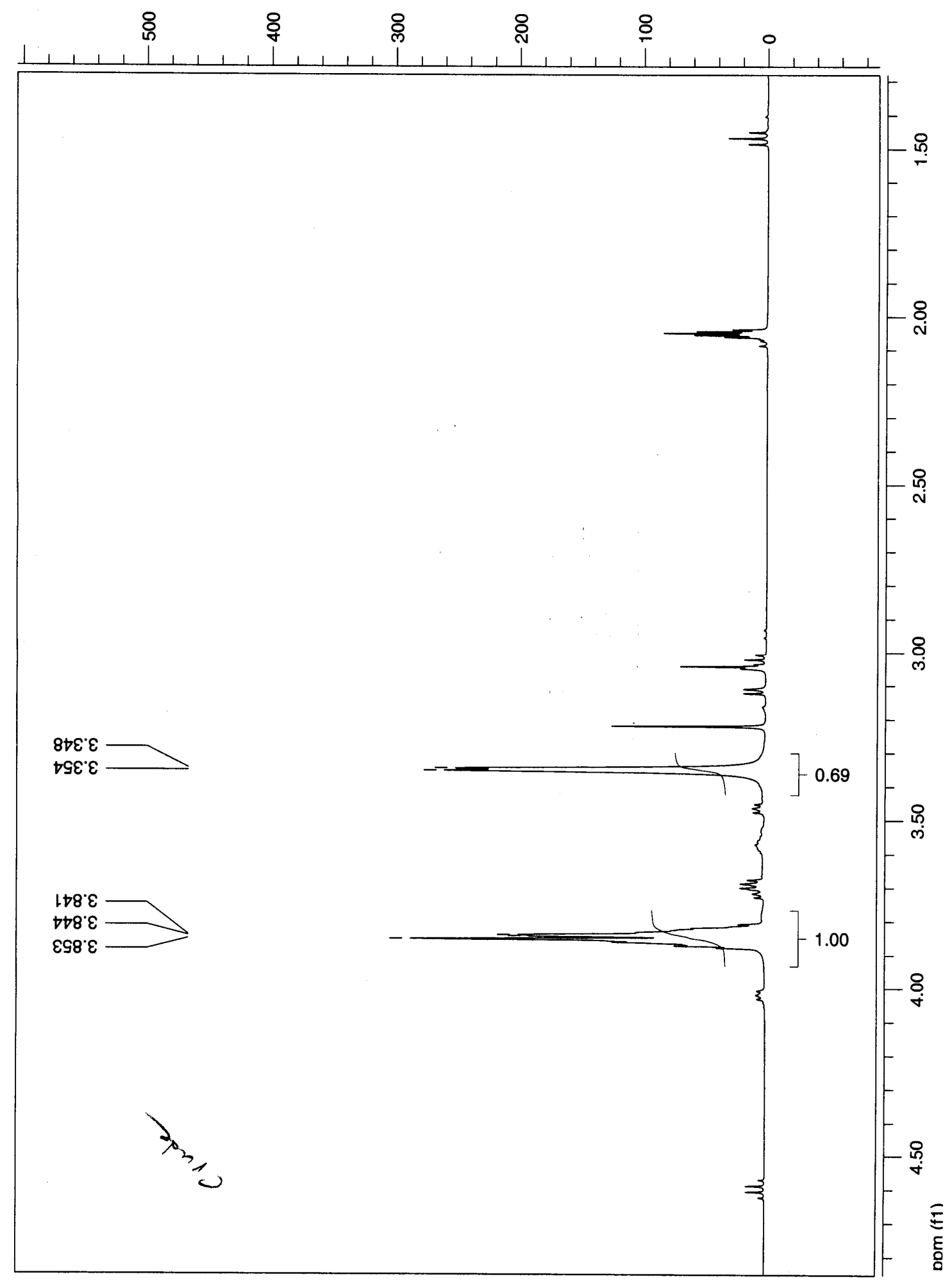

\section{7, H-NMR}




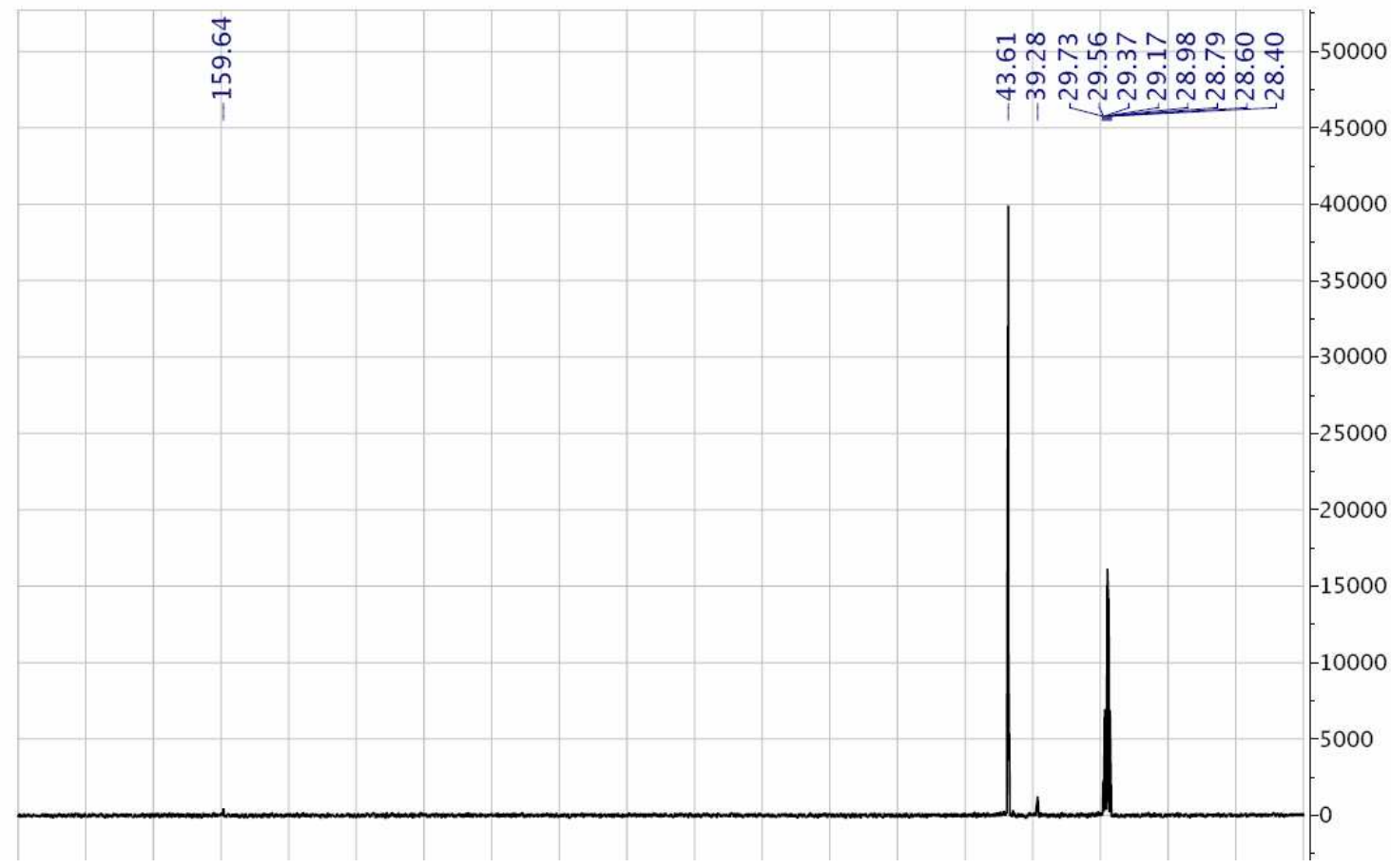

\section{7, C-NMR}




\section{5a, X-ray table}

PREVIEW

24 Aug 2006

LOUIS J. FARRUGIA

E-mail:louis@chem.gla.ac.uk

\section{Experimental}

Crystal data

$\mathrm{C}_{12} \mathrm{H}_{18} \mathrm{~N}_{6} \mathrm{O}_{2} \cdot \mathrm{F}_{6} \mathrm{P}$

$\lambda=0.71073 \AA$

$M_{r}=423.29$

Mo $K \alpha$ radiation

Monoclinic

$P 2_{1} / c$

$a=6.1372(10) \AA$

$\lambda=0.7107 \AA$

Cell parameters from 4304 reflections

$b=22.607$ (4) §

$\theta=3.39-22.95^{\circ}$

$c=12.0984(19) \AA$

$\mu=0.256 \mathrm{~mm}^{-1}$

$\beta=102.067(9)^{\circ}$

$T=100(2) \mathrm{K}$

$V=1641.4(5) \AA^{3}$

Prism

$Z=4$

$D_{x}=1.713 \mathrm{Mg} \mathrm{m}^{-3}$

Colourless

$0.46 \times 0.08 \times 0.06 \mathrm{~mm}$

$D_{m}$ not measured

Crystal source: synthesis as described

Data collection

BRUKER APPEX-II CCD diffractometer

1699 reflections with

Four-circle diffractometer

$>2 \operatorname{sigma}(I)$

$\omega$ and phi scans

$R_{\text {int }}=0.1171$

Absorption correction:

multi-scan BRUKER SADABS

$\theta_{\max }=23.25^{\circ}$

$h=-6 \rightarrow 6$

$k=0 \rightarrow 25$

Please give reference

$T_{\min }=0.8913, T_{\max }=0.9848$

$l=0 \rightarrow 13$

23665 measured reflections

? standard reflections

2350 independent reflections

every? reflections

intensity decay: ?\% 
Refinement

Refinement on $F^{2}$

$R(F)=0.0686$

$w R\left(F^{2}\right)=0.1857$

$S=0.988$

2350 reflections

304 parameters

$\mathrm{H}$-atom parameters constrained $w=1 /\left[\sigma^{2}\left(F_{o}^{2}\right)+(0.1317 P)^{2}+0.0000 P\right]$ where $P=\left(F_{o}^{2}+2 F_{c}^{2}\right) / 3$
$(\Delta / \sigma)_{\max }=0$

$\Delta \rho_{\max }=0.432 \mathrm{e}^{-3}$

$\Delta \rho_{\min }=-0.449$ e $\AA^{-3}$

Extinction correction: SHELXL

Extinction coefficient: 0.019 (5)

Scattering factors from International Tables

for Crystallography (Vol. C) 
Table 1. Fractional atomic coordinates and equivalent isotropic displacement parameters $\left(\AA^{2}\right)$

\begin{tabular}{|c|c|c|c|c|c|}
\hline & & $U_{\text {eq }}=(1 /$ & $\Sigma_{j} U^{i j} a^{i} a^{j} \mathbf{a}_{i} \cdot \mathbf{a}_{j}$ & & \\
\hline & Occupancy & $x$ & $y$ & $z$ & $U_{\text {eq }}$ \\
\hline N1 & 1 & $0.2637(7)$ & $0.09246(17)$ & $0.6003(3)$ & $0.0232(11)$ \\
\hline $\mathrm{N} 2$ & 1 & $0.4708(7)$ & $0.06605(19)$ & $0.6289(4)$ & $0.0282(11)$ \\
\hline N3 & 1 & $0.5511(7)$ & $0.06601(17)$ & $0.5371(3)$ & $0.0224(10)$ \\
\hline $\mathrm{C} 4$ & 1 & $0.4019(8)$ & $0.09336(19)$ & $0.4461(4)$ & $0.0205(12)$ \\
\hline N5 & 1 & $0.4406(7)$ & $0.10146(17)$ & $0.3431(4)$ & $0.0286(11)$ \\
\hline $\mathrm{C} 6$ & 1 & $0.2699(9)$ & $0.1294(2)$ & $0.2757(5)$ & $0.0328(14)$ \\
\hline $\mathrm{C} 7$ & 1 & $0.0756(9)$ & $0.1481(2)$ & $0.3091(5)$ & $0.0321(14)$ \\
\hline $\mathrm{C} 8$ & 1 & $0.0414(9)$ & $0.1387(2)$ & $0.4153(5)$ & $0.0271(13)$ \\
\hline C9 & 1 & $0.2200(8)$ & $0.10994(19)$ & $0.4881(4)$ & $0.0219(12)$ \\
\hline O10 & 1 & $0.7403(6)$ & $0.04504(15)$ & $0.5343(3)$ & $0.0315(10)$ \\
\hline $\mathrm{C} 11$ & 1 & $0.1456(8)$ & $0.1058(2)$ & $0.6834(4)$ & $0.0244(13)$ \\
\hline N12 & 1 & $0.0663(7)$ & $0.16010(19)$ & $0.6844(4)$ & $0.0327(12)$ \\
\hline $\mathrm{C} 13$ & 1 & $-0.1297(10)$ & $0.1751(3)$ & $0.7294(6)$ & $0.0523(18)$ \\
\hline $\mathrm{C} 14$ & 1 & $0.1813(11)$ & $0.2119(2)$ & $0.6475(6)$ & $0.0447(17)$ \\
\hline N15 & 1 & $0.1193(7)$ & $0.06379(18)$ & 0.7556 & $0.0282(11)$ \\
\hline $\mathrm{C} 16$ & 1 & $0.1229(9)$ & $-0.0001(2)$ & 0.7291 & $0.0302(13)$ \\
\hline $\mathrm{C} 17$ & 1 & $0.2786(10)$ & $-0.0314(3)$ & $0.8245(5)$ & $0.0411(16)$ \\
\hline O18 & 1 & $0.2159(6)$ & $-0.0212(2)$ & $0.9299(3)$ & $0.0466(12)$ \\
\hline $\mathrm{C} 19$ & 1 & $0.2307(10)$ & $0.0406(3)$ & $0.9557(5)$ & $0.0449(16)$ \\
\hline $\mathrm{C} 20$ & 1 & $0.0726(9)$ & $0.0759(2)$ & 0.8688 & $0.0338(14)$ \\
\hline P31A & 0.67 & $0.5454(5)$ & $0.21756(18)$ & $0.9486(3)$ & $0.0360(8)$ \\
\hline F32A & 0.67 & $0.8061(9)$ & $0.2055(2)$ & $0.9883(5)$ & $0.0447(14)$ \\
\hline F33A & 0.67 & $0.5377(16)$ & $0.1552(7)$ & $0.8792(10)$ & $0.029(2)$ \\
\hline F34A & 0.67 & $0.5868(9)$ & $0.2502(2)$ & $0.8375(5)$ & $0.0470(14)$ \\
\hline F35A & 0.67 & $0.2816(14)$ & $0.2297(4)$ & $0.9103(7)$ & $0.047(2)$ \\
\hline F36A & 0.67 & $0.5003(10)$ & $0.1859(2)$ & $1.0592(6)$ & $0.0475(15)$ \\
\hline F37A & 0.67 & $0.5736(9)$ & $0.28012(19)$ & $1.0141(4)$ & $0.0484(14)$ \\
\hline P31B & 0.33 & $0.5704(8)$ & $0.1862(3)$ & $0.9923(6)$ & $0.0242(11)$ \\
\hline F36B & 0.33 & $0.4696(15)$ & $0.1319(4)$ & $1.0483(7)$ & $0.033(2)$ \\
\hline F32B & 0.33 & $0.8133(14)$ & $0.1587(4)$ & $1.0239(8)$ & $0.035(2)$ \\
\hline F37B & 0.33 & $0.6170(17)$ & $0.2199(4)$ & $1.1105(8)$ & $0.037(2)$ \\
\hline F34B & 0.33 & $0.665(2)$ & $0.2425(5)$ & $0.9330(11)$ & $0.050(3)$ \\
\hline F35B & 0.33 & $0.329(2)$ & $0.2135(7)$ & $0.9550(14)$ & $0.040(4)$ \\
\hline F33B & 0.33 & $0.479(5)$ & $0.1547(15)$ & $0.875(2)$ & $0.029(2)$ \\
\hline
\end{tabular}

Table 2. Anisotropic displacement parameters $\left(\AA^{2}\right)$

$$
\begin{array}{llllll}
U_{11} & U_{22} & U_{33} & U_{12} & U_{13} & U_{23}
\end{array}
$$




\begin{tabular}{|c|c|c|c|c|c|c|}
\hline N1 & $0.022(3)$ & $0.034(2)$ & $0.010(2)$ & $-0.0003(18)$ & $-0.0040(19)$ & $0.0006(18)$ \\
\hline $\mathrm{N} 2$ & 0.025 & $0.045(3)$ & $0.011(3)$ & $0.001(2)$ & $-0.004(2)$ & $-0.001(2)$ \\
\hline N3 & $0.019(3)$ & $0.033(2)$ & $0.011(3)$ & $-0.0004(19)$ & $-0.0061(19)$ & $-0.0003(18)$ \\
\hline $\mathrm{C} 4$ & $0.027(3)$ & $0.021(2)$ & $0.010(3)$ & $-0.001(2)$ & $-0.006(2)$ & $0.003(2)$ \\
\hline N5 & $0.037(3)$ & $0.030(2)$ & $0.017(3)$ & $-0.004(2)$ & $0.000(2)$ & $0.0034(19)$ \\
\hline $\mathrm{C} 6$ & $0.044(4)$ & $0.037(3)$ & $0.015(3)$ & $-0.001(3)$ & $-0.002(3)$ & $0.008(2)$ \\
\hline $\mathrm{C} 7$ & $0.031(3)$ & $0.036(3)$ & $0.024(3)$ & $0.004(2)$ & $-0.008(3)$ & $0.018(2)$ \\
\hline $\mathrm{C} 8$ & $0.026(3)$ & $0.031(3)$ & $0.022(3)$ & $0.003(2)$ & $-0.001(2)$ & $0.009(2)$ \\
\hline $\mathrm{C} 9$ & $0.026(3)$ & $0.021(2)$ & $0.015(3)$ & $-0.002(2)$ & $-0.006(2)$ & $0.001(2)$ \\
\hline O10 & $0.026(2)$ & $0.046(2)$ & $0.020(2)$ & $0.0049(17)$ & $-0.0008(17)$ & $0.0051(17)$ \\
\hline $\mathrm{C} 11$ & $0.021(3)$ & $0.038(3)$ & $0.009(3)$ & $-0.008(2)$ & $-0.007(2)$ & $0.000(2)$ \\
\hline N12 & $0.038(3)$ & $0.039(3)$ & $0.021(3)$ & $-0.003(2)$ & $0.005(2)$ & $-0.004(2)$ \\
\hline $\mathrm{C} 13$ & $0.053(4)$ & $0.064(4)$ & $0.045(4)$ & $0.018(3)$ & $0.022(3)$ & $0.011(3)$ \\
\hline $\mathrm{C} 14$ & $0.065(5)$ & $0.034(3)$ & $0.040(4)$ & $-0.009(3)$ & $0.022(3)$ & $-0.011(3)$ \\
\hline N15 & 0.028 & $0.042(2)$ & $0.011(2)$ & $-0.006(2)$ & $-0.0046(19)$ & $0.000(2)$ \\
\hline $\mathrm{C} 16$ & $0.034(3)$ & $0.037(3)$ & $0.016(3)$ & $-0.004(2)$ & $-0.006(2)$ & $0.005(2)$ \\
\hline $\mathrm{C} 17$ & 0.038 & $0.061(4)$ & $0.021(4)$ & $0.006(3)$ & $-0.001(3)$ & $0.020(3)$ \\
\hline O18 & $0.037(3)$ & $0.082(3)$ & $0.018(2)$ & $0.009(2)$ & $-0.0003(19)$ & $0.020(2)$ \\
\hline C19 & $0.032(4)$ & $0.080(5)$ & $0.017(3)$ & $-0.002(3)$ & $-0.008(3)$ & $0.006(3)$ \\
\hline $\mathrm{C} 20$ & $0.028(3)$ & $0.055(3)$ & $0.016(3)$ & $-0.008(3)$ & $-0.002(2)$ & $0.000(3)$ \\
\hline P31A & $0.051(2)$ & $0.0350(17)$ & $0.0236(17)$ & $-0.0011(15)$ & $0.0123(14)$ & $-0.0039(14)$ \\
\hline F32A & 0.045 & $0.052(3)$ & $0.038(3)$ & $-0.005(3)$ & $0.013(3)$ & $-0.014(3)$ \\
\hline F33A & $0.017(7)$ & $0.0403(17)$ & $0.028(2)$ & $-0.003(4)$ & $0.001(4)$ & $-0.0025(15)$ \\
\hline F34A & $0.078(4)$ & $0.040(3)$ & $0.027(3)$ & $0.008(3)$ & $0.023(3)$ & $0.009(2)$ \\
\hline F35A & $0.045(5)$ & $0.052(4)$ & $0.041(6)$ & $0.018(3)$ & 0.008 & $-0.006(4)$ \\
\hline F36A & $0.054(4)$ & $0.063(4)$ & $0.030(4)$ & $0.009(3)$ & $0.018(3)$ & $0.013(3)$ \\
\hline F37A & 0.070 & $0.042(3)$ & $0.035(3)$ & $0.002(2)$ & $0.016(3)$ & $-0.013(2)$ \\
\hline P31B & $0.027(3)$ & $0.025(3)$ & $0.019(3)$ & $-0.007(2)$ & $0.000(2)$ & $-0.005(3)$ \\
\hline F36B & $0.046(6)$ & $0.037(5)$ & $0.014(5)$ & $-0.013(4)$ & $0.001(4)$ & $-0.001(4)$ \\
\hline F32B & $0.036(6)$ & $0.045(6)$ & $0.022(6)$ & $0.001(4)$ & $-0.003(4)$ & $-0.005(4)$ \\
\hline F37B & $0.041(6)$ & $0.035(5)$ & $0.030(6)$ & $0.000(5)$ & $-0.007(5)$ & $-0.018(5)$ \\
\hline F34B & $0.066(9)$ & $0.039(6)$ & $0.041(9)$ & $-0.021(7)$ & $0.002(7)$ & $0.020(6)$ \\
\hline F35B & $0.019(8)$ & $0.047(9)$ & $0.044(11)$ & $0.011(6)$ & $-0.015(7)$ & $-0.009(7)$ \\
\hline F33B & $0.017(7)$ & $0.0403(17)$ & $0.028(2)$ & $-0.003(4)$ & $0.001(4)$ & $-0.0025(15)$ \\
\hline
\end{tabular}




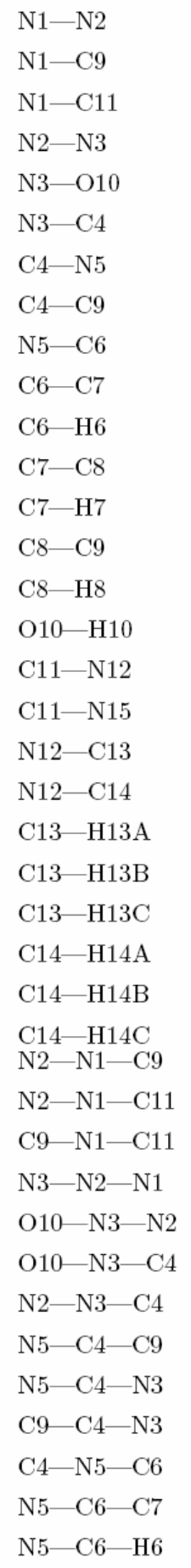

Table 3. Selected geometric parameters $\left(\AA,^{\circ}\right)$

\begin{tabular}{|c|c|c|}
\hline $1.381(6)$ & $\mathrm{N} 15-\mathrm{C} 16$ & $1.482(7)$ \\
\hline $1.385(6)$ & $\mathrm{N} 15-\mathrm{C} 20$ & $1.483(7)$ \\
\hline $1.390(6)$ & $\mathrm{C} 16-\mathrm{C} 17$ & $1.511(7)$ \\
\hline $1.306(6)$ & $\mathrm{C} 16-\mathrm{H} 16 \mathrm{~A}$ & 0.99 \\
\hline $1.261(5)$ & $\mathrm{C} 16-\mathrm{H} 16 \mathrm{~B}$ & 0.99 \\
\hline $1.418(6)$ & $\mathrm{C} 17-\mathrm{O} 18$ & $1.425(7)$ \\
\hline $1.329(6)$ & $\mathrm{C} 17-\mathrm{H} 17 \mathrm{~A}$ & 0.99 \\
\hline $1.372(7)$ & $\mathrm{C} 17-\mathrm{H} 17 \mathrm{~B}$ & 0.99 \\
\hline $1.344(7)$ & $\mathrm{O} 18-\mathrm{C} 19$ & $1.431(7)$ \\
\hline $1.402(8)$ & $\mathrm{C} 19-\mathrm{C} 20$ & $1.502(8)$ \\
\hline 0.95 & $\mathrm{C} 19-\mathrm{H} 19 \mathrm{~A}$ & 0.99 \\
\hline $1.361(7)$ & C19-H19B & 0.99 \\
\hline 0.95 & $\mathrm{C} 20-\mathrm{H} 20 \mathrm{~A}$ & 0.99 \\
\hline $1.413(7)$ & $\mathrm{C} 20-\mathrm{H} 20 \mathrm{~B}$ & 0.99 \\
\hline 0.95 & $\mathrm{P} 31 \mathrm{~A}-\mathrm{F} 36 \mathrm{~A}$ & $1.593(6)$ \\
\hline 0.84 & P31A-F32A & $1.595(6)$ \\
\hline $1.321(7)$ & P31A-F34A & $1.600(6)$ \\
\hline $1.324(6)$ & $\mathrm{P} 31 \mathrm{~A}-\mathrm{F} 35 \mathrm{~A}$ & $1.612(8)$ \\
\hline $1.460(7)$ & P31A-F37A & $1.613(5)$ \\
\hline $1.484(7)$ & $\mathrm{P} 31 \mathrm{~A}-\mathrm{F} 33 \mathrm{~A}$ & $1.637(12)$ \\
\hline 0.98 & P31B-F35B & $1.581(13)$ \\
\hline 0.98 & P31B-F33B & $1.59(2)$ \\
\hline 0.98 & $\mathrm{P} 31 \mathrm{~B}-\mathrm{F} 32 \mathrm{~B}$ & $1.587(10)$ \\
\hline 0.98 & P31B-F36B & $1.588(10)$ \\
\hline 0.98 & $\mathrm{P} 31 \mathrm{~B}-\mathrm{F} 37 \mathrm{~B}$ & $1.593(10)$ \\
\hline $\begin{array}{l}0.98 \\
110.4(4)\end{array}$ & $\begin{array}{l}\mathrm{P} 31 \mathrm{~B}-\mathrm{F} 34 \mathrm{~B} \\
\mathrm{C} 7-\mathrm{C} 6-\mathrm{H} 6\end{array}$ & $\begin{array}{l}1.628(11) \\
117.7\end{array}$ \\
\hline $120.3(4)$ & $\mathrm{C} 8-\mathrm{C} 7-\mathrm{C} 6$ & $122.2(5)$ \\
\hline $128.5(4)$ & $\mathrm{C} 8-\mathrm{C} 7-\mathrm{H} 7$ & 118.9 \\
\hline $106.1(4)$ & $\mathrm{C} 6-\mathrm{C} 7-\mathrm{H} 7$ & 118.9 \\
\hline $122.4(4)$ & $\mathrm{C} 7-\mathrm{C} 8-\mathrm{C} 9$ & $114.1(5)$ \\
\hline $126.3(4)$ & $\mathrm{C} 7-\mathrm{C} 8-\mathrm{H} 8$ & 123 \\
\hline $111.3(4)$ & $\mathrm{C} 9-\mathrm{C} 8-\mathrm{H} 8$ & 123 \\
\hline $128.9(5)$ & $\mathrm{C} 4-\mathrm{C} 9-\mathrm{N} 1$ & $106.2(4)$ \\
\hline $125.1(4)$ & $\mathrm{C} 4-\mathrm{C} 9-\mathrm{C} 8$ & $118.7(5)$ \\
\hline $106.0(4)$ & $\mathrm{N} 1-\mathrm{C} 9-\mathrm{C} 8$ & $135.1(5)$ \\
\hline $111.4(4)$ & $\mathrm{N} 3-\mathrm{O} 10-\mathrm{H} 10$ & 109.5 \\
\hline $124.7(5)$ & $\mathrm{N} 12-\mathrm{C} 11-\mathrm{N} 15$ & $124.4(5)$ \\
\hline 117.7 & $\mathrm{~N} 12-\mathrm{C} 11-\mathrm{N} 1$ & $117.2(4)$ \\
\hline
\end{tabular}




\begin{tabular}{|c|c|}
\hline $\mathrm{N} 15-\mathrm{C} 11-\mathrm{N} 1$ & $118.4(4)$ \\
\hline $\mathrm{C} 11-\mathrm{N} 12-\mathrm{C} 13$ & $123.6(5)$ \\
\hline $\mathrm{C} 11-\mathrm{N} 12-\mathrm{C} 14$ & $122.1(5)$ \\
\hline $\mathrm{C} 13-\mathrm{N} 12-\mathrm{C} 14$ & $114.1(5)$ \\
\hline $\mathrm{N} 12-\mathrm{C} 13-\mathrm{H} 13 \mathrm{~A}$ & 109.5 \\
\hline $\mathrm{N} 12-\mathrm{C} 13-\mathrm{H} 13 \mathrm{~B}$ & 109.5 \\
\hline $\mathrm{H} 13 \mathrm{~A}-\mathrm{C} 13-\mathrm{H} 13 \mathrm{~B}$ & 109.5 \\
\hline $\mathrm{N} 12-\mathrm{C} 13-\mathrm{H} 13 \mathrm{C}$ & 109.5 \\
\hline $\mathrm{H} 13 \mathrm{~A}-\mathrm{C} 13-\mathrm{H} 13 \mathrm{C}$ & 109.5 \\
\hline $\mathrm{H} 13 \mathrm{~B}-\mathrm{C} 13-\mathrm{H} 13 \mathrm{C}$ & 109.5 \\
\hline $\mathrm{N} 12-\mathrm{C} 14-\mathrm{H} 14 \mathrm{~A}$ & 109.5 \\
\hline $\mathrm{N} 12-\mathrm{C} 14-\mathrm{H} 14 \mathrm{~B}$ & 109.5 \\
\hline $\mathrm{H} 14 \mathrm{~A}-\mathrm{C} 14-\mathrm{H} 14 \mathrm{~B}$ & 109.5 \\
\hline $\mathrm{N} 12-\mathrm{C} 14-\mathrm{H} 14 \mathrm{C}$ & 109.5 \\
\hline $\mathrm{H} 14 \mathrm{~A}-\mathrm{C} 14-\mathrm{H} 14 \mathrm{C}$ & 109.5 \\
\hline $\mathrm{H} 14 \mathrm{~B}-\mathrm{C} 14-\mathrm{H} 14 \mathrm{C}$ & 109.5 \\
\hline $\mathrm{C} 11-\mathrm{N} 15-\mathrm{C} 16$ & $123.3(4)$ \\
\hline $\mathrm{C} 11-\mathrm{N} 15-\mathrm{C} 20$ & $123.4(4)$ \\
\hline $\mathrm{C} 16-\mathrm{N} 15-\mathrm{C} 20$ & $113.3(4)$ \\
\hline $\mathrm{N} 15-\mathrm{C} 16-\mathrm{C} 17$ & $109.1(4)$ \\
\hline $\mathrm{N} 15-\mathrm{C} 16-\mathrm{H} 16 \mathrm{~A}$ & 109.9 \\
\hline $\mathrm{C} 17-\mathrm{C} 16-\mathrm{H} 16 \mathrm{~A}$ & 109.9 \\
\hline $\mathrm{N} 15-\mathrm{C} 16-\mathrm{H} 16 \mathrm{~B}$ & 109.9 \\
\hline $\mathrm{C} 17-\mathrm{C} 16-\mathrm{H} 16 \mathrm{~B}$ & 109.9 \\
\hline $\mathrm{H} 16 \mathrm{~A}-\mathrm{C} 16-\mathrm{H} 16 \mathrm{~B}$ & 108.3 \\
\hline $\mathrm{O} 18-\mathrm{C} 17-\mathrm{C} 16$ & $111.2(5)$ \\
\hline $\mathrm{O} 18-\mathrm{C} 17-\mathrm{H} 17 \mathrm{~A}$ & 109.4 \\
\hline $\mathrm{C} 16-\mathrm{C} 17-\mathrm{H} 17 \mathrm{~A}$ & 109.4 \\
\hline $\mathrm{O} 18-\mathrm{C} 17-\mathrm{H} 17 \mathrm{~B}$ & 109.4 \\
\hline $\mathrm{C} 16-\mathrm{C} 17-\mathrm{H} 17 \mathrm{~B}$ & 109.4 \\
\hline $\mathrm{H} 17 \mathrm{~A}-\mathrm{C} 17-\mathrm{H} 17 \mathrm{~B}$ & 108 \\
\hline $\mathrm{C} 17-\mathrm{O} 18-\mathrm{C} 19$ & $109.7(4)$ \\
\hline $\mathrm{O} 18-\mathrm{C} 19-\mathrm{C} 20$ & $111.4(5)$ \\
\hline $\mathrm{O} 18-\mathrm{C} 19-\mathrm{H} 19 \mathrm{~A}$ & 109.4 \\
\hline $\mathrm{C} 20-\mathrm{C} 19-\mathrm{H} 19 \mathrm{~A}$ & 109.4 \\
\hline $\mathrm{O} 18-\mathrm{C} 19-\mathrm{H} 19 \mathrm{~B}$ & 109.4 \\
\hline $\mathrm{C} 20-\mathrm{C} 19-\mathrm{H} 19 \mathrm{~B}$ & 109.4 \\
\hline
\end{tabular}

\begin{tabular}{|c|c|}
\hline $\mathrm{H} 19 \mathrm{~A}-\mathrm{C} 19-\mathrm{H} 19 \mathrm{~B}$ & 108 \\
\hline $\mathrm{N} 15-\mathrm{C} 20-\mathrm{C} 19$ & $109.1(5)$ \\
\hline $\mathrm{N} 15-\mathrm{C} 20-\mathrm{H} 20 \mathrm{~A}$ & 109.9 \\
\hline $\mathrm{C} 19-\mathrm{C} 20-\mathrm{H} 20 \mathrm{~A}$ & 109.9 \\
\hline $\mathrm{N} 15-\mathrm{C} 20-\mathrm{H} 20 \mathrm{~B}$ & 109.9 \\
\hline $\mathrm{C} 19-\mathrm{C} 20-\mathrm{H} 20 \mathrm{~B}$ & 109.9 \\
\hline $\mathrm{H} 20 \mathrm{~A}-\mathrm{C} 20-\mathrm{H} 20 \mathrm{~B}$ & 108.3 \\
\hline $\mathrm{F} 36 \mathrm{~A}-\mathrm{P} 31 \mathrm{~A}-\mathrm{F} 32 \mathrm{~A}$ & $90.5(4)$ \\
\hline F $36 \mathrm{~A}-\mathrm{P} 31 \mathrm{~A}-\mathrm{F} 34 \mathrm{~A}$ & $178.9(4)$ \\
\hline $\mathrm{F} 32 \mathrm{~A}-\mathrm{P} 31 \mathrm{~A}-\mathrm{F} 34 \mathrm{~A}$ & $90.4(4)$ \\
\hline $\mathrm{F} 36 \mathrm{~A}-\mathrm{P} 31 \mathrm{~A}-\mathrm{F} 35 \mathrm{~A}$ & $88.7(4)$ \\
\hline $\mathrm{F} 32 \mathrm{~A}-\mathrm{P} 31 \mathrm{~A}-\mathrm{F} 35 \mathrm{~A}$ & $179.2(5)$ \\
\hline $\mathrm{F} 34 \mathrm{~A}-\mathrm{P} 31 \mathrm{~A}-\mathrm{F} 35 \mathrm{~A}$ & $90.3(4)$ \\
\hline F36A-P31A-F37A & $90.1(3)$ \\
\hline $\mathrm{F} 32 \mathrm{~A}-\mathrm{P} 31 \mathrm{~A}-\mathrm{F} 37 \mathrm{~A}$ & $90.3(3)$ \\
\hline $\mathrm{F} 34 \mathrm{~A}-\mathrm{P} 31 \mathrm{~A}-\mathrm{F} 37 \mathrm{~A}$ & $89.3(4)$ \\
\hline $\mathrm{F} 35 \mathrm{~A}-\mathrm{P} 31 \mathrm{~A}-\mathrm{F} 37 \mathrm{~A}$ & $89.4(4)$ \\
\hline $\mathrm{F} 36 \mathrm{~A}-\mathrm{P} 31 \mathrm{~A}-\mathrm{F} 33 \mathrm{~A}$ & $93.0(7)$ \\
\hline $\mathrm{F} 32 \mathrm{~A}-\mathrm{P} 31 \mathrm{~A}-\mathrm{F} 33 \mathrm{~A}$ & $85.8(4)$ \\
\hline $\mathrm{F} 34 \mathrm{~A}-\mathrm{P} 31 \mathrm{~A}-\mathrm{F} 33 \mathrm{~A}$ & $87.7(6)$ \\
\hline $\mathrm{F} 35 \mathrm{~A}-\mathrm{P} 31 \mathrm{~A}-\mathrm{F} 33 \mathrm{~A}$ & $94.6(5)$ \\
\hline $\mathrm{F} 37 \mathrm{~A}-\mathrm{P} 31 \mathrm{~A}-\mathrm{F} 33 \mathrm{~A}$ & $175.0(5)$ \\
\hline $\mathrm{F} 35 \mathrm{~B}-\mathrm{P} 31 \mathrm{~B}-\mathrm{F} 33 \mathrm{~B}$ & $77.7(12)$ \\
\hline $\mathrm{F} 35 \mathrm{~B}-\mathrm{P} 31 \mathrm{~B}-\mathrm{F} 32 \mathrm{~B}$ & $177.4(9)$ \\
\hline $\mathrm{F} 33 \mathrm{~B}-\mathrm{P} 31 \mathrm{~B}-\mathrm{F} 32 \mathrm{~B}$ & $100.2(12)$ \\
\hline $\mathrm{F} 35 \mathrm{~B}-\mathrm{P} 31 \mathrm{~B}-\mathrm{F} 36 \mathrm{~B}$ & $89.9(7)$ \\
\hline $\mathrm{F} 33 \mathrm{~B}-\mathrm{P} 31 \mathrm{~B}-\mathrm{F} 36 \mathrm{~B}$ & $86.6(13)$ \\
\hline $\mathrm{F} 32 \mathrm{~B}-\mathrm{P} 31 \mathrm{~B}-\mathrm{F} 36 \mathrm{~B}$ & $91.4(6)$ \\
\hline F35B-P31B-F37B & $92.9(8)$ \\
\hline $\mathrm{F} 33 \mathrm{~B}-\mathrm{P} 31 \mathrm{~B}-\mathrm{F} 37 \mathrm{~B}$ & $170.0(12)$ \\
\hline $\mathrm{F} 32 \mathrm{~B}-\mathrm{P} 31 \mathrm{~B}-\mathrm{F} 37 \mathrm{~B}$ & $89.3(5)$ \\
\hline F36B-P31B-F37B & $90.0(7)$ \\
\hline $\mathrm{F} 35 \mathrm{~B}-\mathrm{P} 31 \mathrm{~B}-\mathrm{F} 34 \mathrm{~B}$ & $88.2(8)$ \\
\hline F33B-P31B-F34B & $92.6(14)$ \\
\hline $\mathrm{F} 32 \mathrm{~B}-\mathrm{P} 31 \mathrm{~B}-\mathrm{F} 34 \mathrm{~B}$ & $90.4(6)$ \\
\hline $\mathrm{F} 36 \mathrm{~B}-\mathrm{P} 31 \mathrm{~B}-\mathrm{F} 34 \mathrm{~B}$ & $178.1(7)$ \\
\hline $\mathrm{F} 37 \mathrm{~B}-\mathrm{P} 31 \mathrm{~B}-\mathrm{F} 34 \mathrm{~B}$ & $90.5(6)$ \\
\hline
\end{tabular}




\begin{tabular}{|c|c|c|c|}
\hline $\mathrm{C} 9-\mathrm{N} 1-\mathrm{N} 2-\mathrm{N} 3$ & $-1.2(5)$ & $\mathrm{C} 7-\mathrm{C} 8-\mathrm{C} 9-\mathrm{N} 1$ & $178.5(5)$ \\
\hline $\mathrm{C} 11-\mathrm{N} 1-\mathrm{N} 2-\mathrm{N} 3$ & $-172.1(4)$ & $\mathrm{N} 2-\mathrm{N} 1-\mathrm{C} 11-\mathrm{N} 12$ & $129.7(5)$ \\
\hline $\mathrm{N} 1-\mathrm{N} 2-\mathrm{N} 3-\mathrm{O} 10$ & $179.7(4)$ & $\mathrm{C} 9-\mathrm{N} 1-\mathrm{C} 11-\mathrm{N} 12$ & $-39.4(7)$ \\
\hline $\mathrm{N} 1-\mathrm{N} 2-\mathrm{N} 3-\mathrm{C} 4$ & $1.2(5)$ & $\mathrm{N} 2-\mathrm{N} 1-\mathrm{C} 11-\mathrm{N} 15$ & $-50.6(6)$ \\
\hline $\mathrm{O} 10-\mathrm{N} 3-\mathrm{C} 4-\mathrm{N} 5$ & $-0.5(7)$ & $\mathrm{C} 9-\mathrm{N} 1-\mathrm{C} 11-\mathrm{N} 15$ & $140.4(5)$ \\
\hline $\mathrm{N} 2-\mathrm{N} 3-\mathrm{C} 4-\mathrm{N} 5$ & $177.9(4)$ & $\mathrm{N} 15-\mathrm{C} 11-\mathrm{N} 12-\mathrm{C} 13$ & $-26.5(8)$ \\
\hline $\mathrm{O} 10-\mathrm{N} 3-\mathrm{C} 4-\mathrm{C} 9$ & $-179.1(4)$ & $\mathrm{N} 1-\mathrm{C} 11-\mathrm{N} 12-\mathrm{C} 13$ & $153.3(5)$ \\
\hline $\mathrm{N} 2-\mathrm{N} 3-\mathrm{C} 4-\mathrm{C} 9$ & $-0.7(5)$ & $\mathrm{N} 15-\mathrm{C} 11-\mathrm{N} 12-\mathrm{C} 14$ & $148.6(5)$ \\
\hline $\mathrm{C} 9-\mathrm{C} 4-\mathrm{N} 5-\mathrm{C} 6$ & $-0.8(7)$ & $\mathrm{N} 1-\mathrm{C} 11-\mathrm{N} 12-\mathrm{C} 14$ & $-31.6(7)$ \\
\hline $\mathrm{N} 3-\mathrm{C} 4-\mathrm{N} 5-\mathrm{C} 6$ & $-179.1(4)$ & $\mathrm{N} 12-\mathrm{C} 11-\mathrm{N} 15-\mathrm{C} 16$ & $154.0(5)$ \\
\hline $\mathrm{C} 4-\mathrm{N} 5-\mathrm{C} 6-\mathrm{C} 7$ & $0.4(7)$ & $\mathrm{N} 1-\mathrm{C} 11-\mathrm{N} 15-\mathrm{C} 16$ & $-25.7(7)$ \\
\hline $\mathrm{N} 5-\mathrm{C} 6-\mathrm{C} 7-\mathrm{C} 8$ & $-0.9(8)$ & $\mathrm{N} 12-\mathrm{C} 11-\mathrm{N} 15-\mathrm{C} 20$ & $-22.9(8)$ \\
\hline $\mathrm{C} 6-\mathrm{C} 7-\mathrm{C} 8-\mathrm{C} 9$ & $1.4(7)$ & $\mathrm{N} 1-\mathrm{C} 11-\mathrm{N} 15-\mathrm{C} 20$ & $157.3(4)$ \\
\hline $\mathrm{N} 5-\mathrm{C} 4-\mathrm{C} 9-\mathrm{N} 1$ & $-178.6(5)$ & $\mathrm{C} 11-\mathrm{N} 15-\mathrm{C} 16-\mathrm{C} 17$ & $130.8(5)$ \\
\hline $\mathrm{N} 3-\mathrm{C} 4-\mathrm{C} 9-\mathrm{N} 1$ & $-0.1(5)$ & $\mathrm{C} 20-\mathrm{N} 15-\mathrm{C} 16-\mathrm{C} 17$ & $-52.0(6)$ \\
\hline $\mathrm{N} 5-\mathrm{C} 4-\mathrm{C} 9-\mathrm{C} 8$ & $1.5(7)$ & $\mathrm{N} 15-\mathrm{C} 16-\mathrm{C} 17-\mathrm{O} 18$ & $56.3(6)$ \\
\hline $\mathrm{N} 3-\mathrm{C} 4-\mathrm{C} 9-\mathrm{C} 8$ & -180.0 & $\mathrm{C} 16-\mathrm{C} 17-\mathrm{O} 18-\mathrm{C} 19$ & $-62.1(6)$ \\
\hline $\mathrm{N} 2-\mathrm{N} 1-\mathrm{C} 9-\mathrm{C} 4$ & $0.8(5)$ & $\mathrm{C} 17-\mathrm{O} 18-\mathrm{C} 19-\mathrm{C} 20$ & $62.4(6)$ \\
\hline $\mathrm{C} 11-\mathrm{N} 1-\mathrm{C} 9-\mathrm{C} 4$ & $170.7(4)$ & $\mathrm{C} 11-\mathrm{N} 15-\mathrm{C} 20-\mathrm{C} 19$ & $-130.6(5)$ \\
\hline $\mathrm{N} 2-\mathrm{N} 1-\mathrm{C} 9-\mathrm{C} 8$ & $-179.3(5)$ & $\mathrm{C} 16-\mathrm{N} 15-\mathrm{C} 20-\mathrm{C} 19$ & $52.1(6)$ \\
\hline $\mathrm{C} 11-\mathrm{N} 1-\mathrm{C} 9-\mathrm{C} 8$ & $-9.4(8)$ & $\mathrm{O} 18-\mathrm{C} 19-\mathrm{C} 20-\mathrm{N} 15$ & $-56.5(6)$ \\
\hline $\mathrm{C} 7-\mathrm{C} 8-\mathrm{C} 9-\mathrm{C} 4$ & $-1.6(6)$ & & \\
\hline
\end{tabular}




\title{
5b, X-ray Table
}

PREVIEW

21 Aug 2006

\author{
Louis J. FARrugia
}

.E-mail: louis@chem.gla.ac.uk

\section{Experimental}

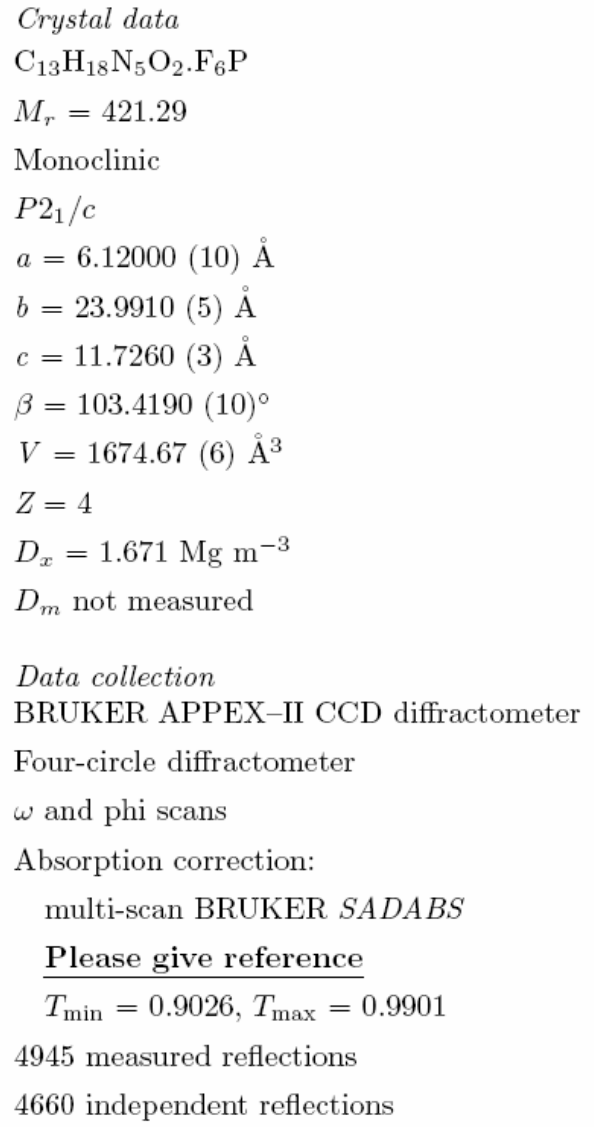

? standard reflections every? reflections

intensity decay: ?\% 
Refinement

Refinement on $F^{2}$

$(\Delta / \sigma)_{\max }=0.001$

$R(F)=0.0412$

$\Delta \rho_{\max }=0.349{\mathrm{e} \AA^{-3}}^{-3}$

$w R\left(F^{2}\right)=0.0978$

$\Delta \rho_{\min }=-0.406$ e $\AA^{-3}$

$S=1.065$

Extinction correction: SHELXL

4945 reflections

Extinction coefficient: 0.0005 (5)

247 parameters

Scattering factors from International Tables

$\mathrm{H}$-atom parameters constrained

$w=1 /\left[\sigma^{2}\left(F_{o}^{2}\right)+(0.0472 P)^{2}+0.2196 P\right]$

for Crystallography (Vol. C)

where $P=\left(F_{o}^{2}+2 F_{c}^{2}\right) / 3$ 
Table 1. Fractional atomic coordinates and equivalent isotropic displacement parameters $\left(\AA^{2}\right)$

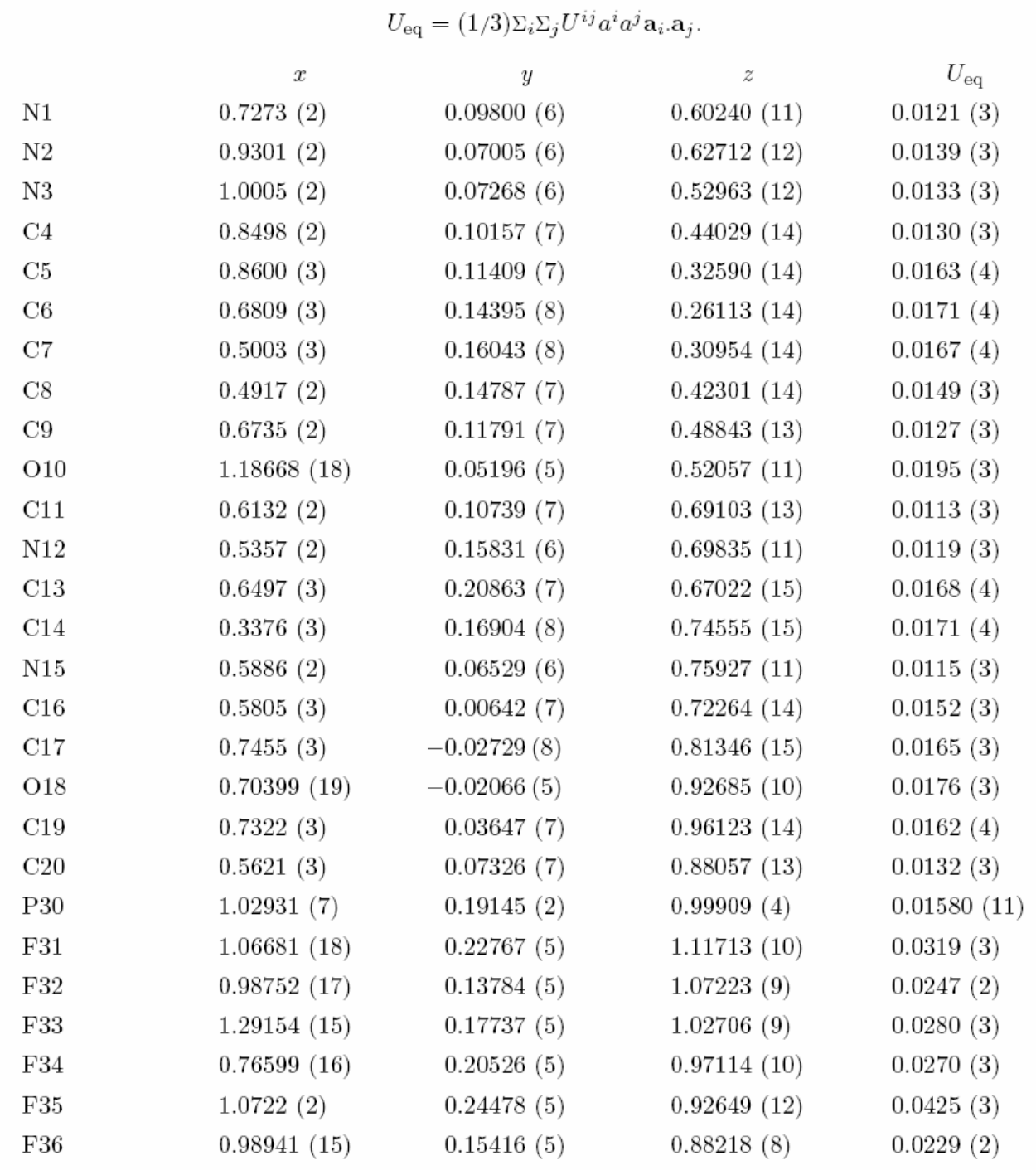

Table 2. Anisotropic displacement parameters $\left(\AA^{2}\right)$

$\begin{array}{llllllr}\mathrm{N} 1 & 0.0113(5) & 0.0147(8) & 0.0106(6) & 0.0030(5) & 0.0036(5) & 0.0032(5) \\ \mathrm{N} 2 & 0.0119(6) & 0.0149(8) & 0.0150(6) & 0.0028(5) & 0.0033(5) & -0.0001(5) \\ \mathrm{N} 3 & 0.0120(6) & 0.0132(8) & 0.0152(6) & 0.0010(5) & 0.0044(5) & -0.0010(5) \\ \mathrm{C} 4 & 0.0122(6) & 0.0123(9) & 0.0145(7) & -0.0015(6) & 0.0032(6) & -0.0010(6) \\ \mathrm{C} 5 & 0.0170(7) & 0.0189(11) & 0.0150(8) & -0.0036(6) & 0.0079(6) & -0.0022(6) \\ \mathrm{C} 6 & 0.0212(8) & 0.0190(11) & 0.0120(7) & -0.0042(7) & 0.0058(6) & 0.0002(6) \\ \mathrm{C} 7 & 0.0172(7) & 0.0173(10) & 0.0143(7) & -0.0013(6) & 0.0011(6) & 0.0021(6)\end{array}$




$\begin{array}{llllrrr}\text { C8 } & 0.0126(7) & 0.0171(10) & 0.0151(7) & 0.0001(6) & 0.0034(6) & 0.0008(6) \\ \text { C9 } & 0.0138(7) & 0.0137(10) & 0.0110(7) & -0.0021(6) & 0.0036(6) & 0.0006(6) \\ \text { O10 } & 0.0128(5) & 0.0241(8) & 0.0233(6) & 0.0056(5) & 0.0074(5) & -0.0005(5) \\ \text { C11 } & 0.0089(6) & 0.0151(10) & 0.0098(7) & -0.0008(6) & 0.0020(5) & 0.0001(6) \\ \text { N12 } & 0.0125(6) & 0.0114(8) & 0.0127(6) & 0.0007(5) & 0.0044(5) & 0.0010(5) \\ \text { C13 } & 0.0205(8) & 0.0110(10) & 0.0194(8) & -0.0015(6) & 0.0057(6) & 0.0019(7) \\ \text { C14 } & 0.0137(7) & 0.0178(10) & 0.0215(8) & 0.0043(6) & 0.0079(6) & 0.0024(7) \\ \text { N15 } & 0.0146(6) & 0.0088(8) & 0.0111(6) & -0.0004(5) & 0.0032(5) & 0.0002(5) \\ \text { C16 } & 0.0193(8) & 0.0105(10) & 0.0158(7) & -0.0017(6) & 0.0038(6) & -0.0005(6) \\ \text { C17 } & 0.0183(7) & 0.0123(10) & 0.0197(8) & 0.0015(6) & 0.0063(6) & 0.0013(7) \\ \text { O18 } & 0.0210(6) & 0.0138(7) & 0.0190(6) & 0.0008(5) & 0.0067(5) & 0.0042(5) \\ \text { C19 } & 0.0174(7) & 0.0179(11) & 0.0134(7) & 0.0016(6) & 0.0037(6) & 0.0034(6) \\ \text { C20 } & 0.0170(7) & 0.0132(10) & 0.0108(7) & 0.0004(6) & 0.0057(6) & 0.0010(6) \\ \text { P30 } & 0.01194(18) & 0.0182(3) & 0.0166(2) & -0.00174(16) & 0.00211(15) & -0.00217(18) \\ \text { F31 } & 0.0260(5) & 0.0313(7) & 0.0343(6) & 0.0011(5) & -0.0010(5) & -0.0207(5) \\ \text { F32 } & 0.0333(6) & 0.0235(7) & 0.0194(5) & -0.0021(5) & 0.0105(4) & 0.0006(4) \\ \text { F33 } & 0.0121(4) & 0.0479(8) & 0.0225(5) & 0.0015(4) & 0.0013(4) & -0.0048(5) \\ \text { F34 } & 0.0149(4) & 0.0332(7) & 0.0309(6) & 0.0047(4) & 0.0013(4) & -0.0093(5) \\ \text { F35 } & 0.0412(7) & 0.0313(8) & 0.0510(8) & -0.0121(6) & 0.0028(6) & 0.0176(6) \\ \text { F36 } & 0.0175(4) & 0.0377(7) & 0.0140(5) & -0.0014(4) & 0.0047(4) & -0.0057(4)\end{array}$


Table 3. Selected geometric parameters $\left(\AA,^{\circ}\right)$

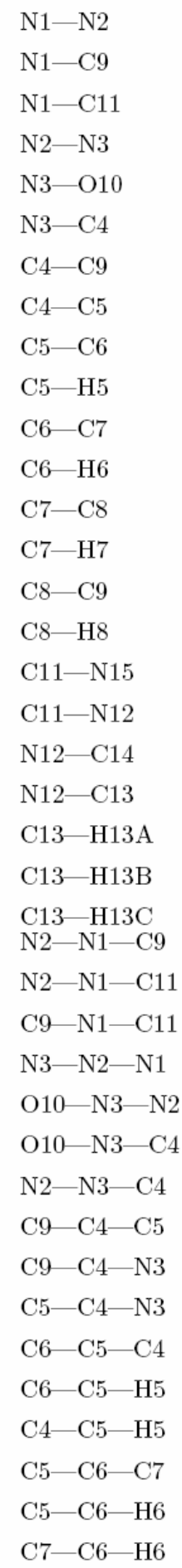

$1.3811(17)$
$1.3850(19)$
$1.3982(19)$
$1.3137(18)$
$1.2697(16)$
$1.407(2)$
$1.385(2)$
$1.390(2)$
$1.380(2)$
0.95
$1.411(2)$
0.95
$1.377(2)$
0.95
$1.395(2)$
0.95
$1.319(2)$
$1.320(2)$
$1.4677(19)$
$1.469(2)$
0.98
0.98
0.98
$111.76(12)$
$120.11(12)$
$127.87(13)$
$104.74(12)$
$122.42(13)$
$125.19(13)$
$112.38(12)$
$123.06(15)$
$105.90(14)$
$131.03(14)$
$115.81(15)$
122.1
122.1
$121.34(15)$
119.3
119.3

$\mathrm{C} 14-\mathrm{H} 14 \mathrm{~A}$

0.98

0.98

$\mathrm{C} 14-\mathrm{H} 14 \mathrm{C} \quad 0.98$

$\mathrm{N} 15-\mathrm{C} 16$

$1.474(2)$

1.4809 (19)

$1.519(2)$

0.99

0.99

1.419 (2)

0.99

C17-H17A

0.99

$1.428(2)$

1.517 (2)

0.99

C19-H19A

0.99

0.99

0.99

1.5919 (13)

1.5978 (10)

$1.5990(12)$

$1.6028(10)$

1.6049 (12)

1.6075 (11)

$122.46(15)$

118.8

118.8

116.06 (15)

122

122

105.22 (13)

133.52 (15)

121.26 (15)

125.16 (14)

118.33 (14)

116.51 (14)

121.82 (14)

123.31 (13)

114.66 (13)

109.5 


\begin{tabular}{|c|c|c|c|}
\hline $\mathrm{N} 12-\mathrm{C} 13-\mathrm{H} 13 \mathrm{~B}$ & 109.5 & $\mathrm{O} 18-\mathrm{C} 19-\mathrm{C} 20$ & $111.22(13)$ \\
\hline $\mathrm{H} 13 \mathrm{~A}-\mathrm{C} 13-\mathrm{H} 13 \mathrm{~B}$ & 109.5 & $\mathrm{O} 18-\mathrm{C} 19-\mathrm{H} 19 \mathrm{~A}$ & 109.4 \\
\hline $\mathrm{N} 12-\mathrm{C} 13-\mathrm{H} 13 \mathrm{C}$ & 109.5 & $\mathrm{C} 20-\mathrm{C} 19-\mathrm{H} 19 \mathrm{~A}$ & 109.4 \\
\hline $\mathrm{H} 13 \mathrm{~A}-\mathrm{C} 13-\mathrm{H} 13 \mathrm{C}$ & 109.5 & $\mathrm{O} 18-\mathrm{C} 19-\mathrm{H} 19 \mathrm{~B}$ & 109.4 \\
\hline $\mathrm{H} 13 \mathrm{~B}-\mathrm{C} 13-\mathrm{H} 13 \mathrm{C}$ & 109.5 & $\mathrm{C} 20-\mathrm{C} 19-\mathrm{H} 19 \mathrm{~B}$ & 109.4 \\
\hline $\mathrm{N} 12-\mathrm{C} 14-\mathrm{H} 14 \mathrm{~A}$ & 109.5 & $\mathrm{H} 19 \mathrm{~A}-\mathrm{C} 19-\mathrm{H} 19 \mathrm{~B}$ & 108 \\
\hline $\mathrm{N} 12-\mathrm{C} 14-\mathrm{H} 14 \mathrm{~B}$ & 109.5 & $\mathrm{~N} 15-\mathrm{C} 20-\mathrm{C} 19$ & $108.14(13)$ \\
\hline $\mathrm{H} 14 \mathrm{~A}-\mathrm{C} 14-\mathrm{H} 14 \mathrm{~B}$ & 109.5 & $\mathrm{~N} 15-\mathrm{C} 20-\mathrm{H} 20 \mathrm{~A}$ & 110.1 \\
\hline $\mathrm{N} 12-\mathrm{C} 14-\mathrm{H} 14 \mathrm{C}$ & 109.5 & $\mathrm{C} 19-\mathrm{C} 20-\mathrm{H} 20 \mathrm{~A}$ & 110.1 \\
\hline $\mathrm{H} 14 \mathrm{~A}-\mathrm{C} 14-\mathrm{H} 14 \mathrm{C}$ & 109.5 & $\mathrm{~N} 15-\mathrm{C} 20-\mathrm{H} 20 \mathrm{~B}$ & 110.1 \\
\hline $\mathrm{H} 14 \mathrm{~B}-\mathrm{C} 14-\mathrm{H} 14 \mathrm{C}$ & 109.5 & $\mathrm{C} 19-\mathrm{C} 20-\mathrm{H} 20 \mathrm{~B}$ & 110.1 \\
\hline $\mathrm{C} 11-\mathrm{N} 15-\mathrm{C} 16$ & $123.92(13)$ & $\mathrm{H} 20 \mathrm{~A}-\mathrm{C} 20-\mathrm{H} 20 \mathrm{~B}$ & 108.4 \\
\hline $\mathrm{C} 11-\mathrm{N} 15-\mathrm{C} 20$ & $122.50(14)$ & F35-P30-F33 & $89.84(7)$ \\
\hline $\mathrm{C} 16-\mathrm{N} 15-\mathrm{C} 20$ & $113.58(13)$ & F35-P30-F32 & $179.72(7)$ \\
\hline $\mathrm{N} 15-\mathrm{C} 16-\mathrm{C} 17$ & $109.52(13)$ & F33-P30-F32 & $89.88(6)$ \\
\hline $\mathrm{N} 15-\mathrm{C} 16-\mathrm{H} 16 \mathrm{~A}$ & 109.8 & F35-P30-F34 & $90.42(7)$ \\
\hline $\mathrm{C} 17-\mathrm{C} 16-\mathrm{H} 16 \mathrm{~A}$ & 109.8 & F33-P30-F34 & $179.72(7)$ \\
\hline $\mathrm{N} 15-\mathrm{C} 16-\mathrm{H} 16 \mathrm{~B}$ & 109.8 & F32-P30-F34 & $89.86(6)$ \\
\hline $\mathrm{C} 17-\mathrm{C} 16-\mathrm{H} 16 \mathrm{~B}$ & 109.8 & F35-P30-F31 & $91.02(7)$ \\
\hline $\mathrm{H} 16 \mathrm{~A}-\mathrm{C} 16-\mathrm{H} 16 \mathrm{~B}$ & 108.2 & F33-P30-F31 & $90.09(6)$ \\
\hline $\mathrm{O} 18-\mathrm{C} 17-\mathrm{C} 16$ & $111.27(13)$ & F32-P30-F31 & $88.94(6)$ \\
\hline $\mathrm{O} 18-\mathrm{C} 17-\mathrm{H} 17 \mathrm{~A}$ & 109.4 & F34-P30-F31 & $90.01(6)$ \\
\hline $\mathrm{C} 16-\mathrm{C} 17-\mathrm{H} 17 \mathrm{~A}$ & 109.4 & F35-P30-F36 & $90.14(7)$ \\
\hline $\mathrm{O} 18-\mathrm{C} 17-\mathrm{H} 17 \mathrm{~B}$ & 109.4 & F33-P30-F36 & $90.22(6)$ \\
\hline $\mathrm{C} 16-\mathrm{C} 17-\mathrm{H} 17 \mathrm{~B}$ & 109.4 & F32-P30-F36 & $89.90(6)$ \\
\hline $\mathrm{H} 17 \mathrm{~A}-\mathrm{C} 17-\mathrm{H} 17 \mathrm{~B}$ & 108 & F34-P30-F36 & $89.67(6)$ \\
\hline $\mathrm{C} 17-\mathrm{O} 18-\mathrm{C} 19$ & $109.62(13)$ & F31-P30-F36 & $178.80(7)$ \\
\hline
\end{tabular}




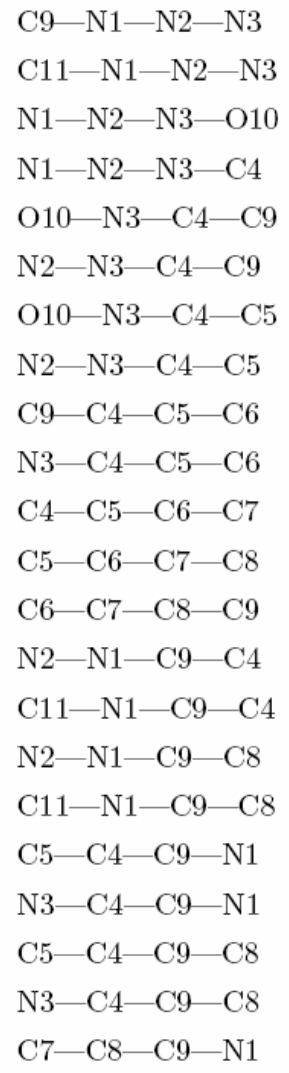

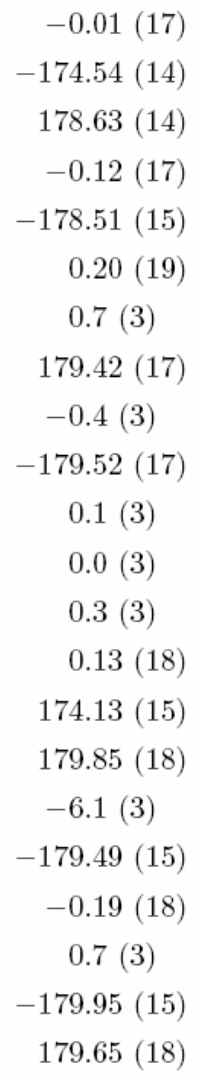

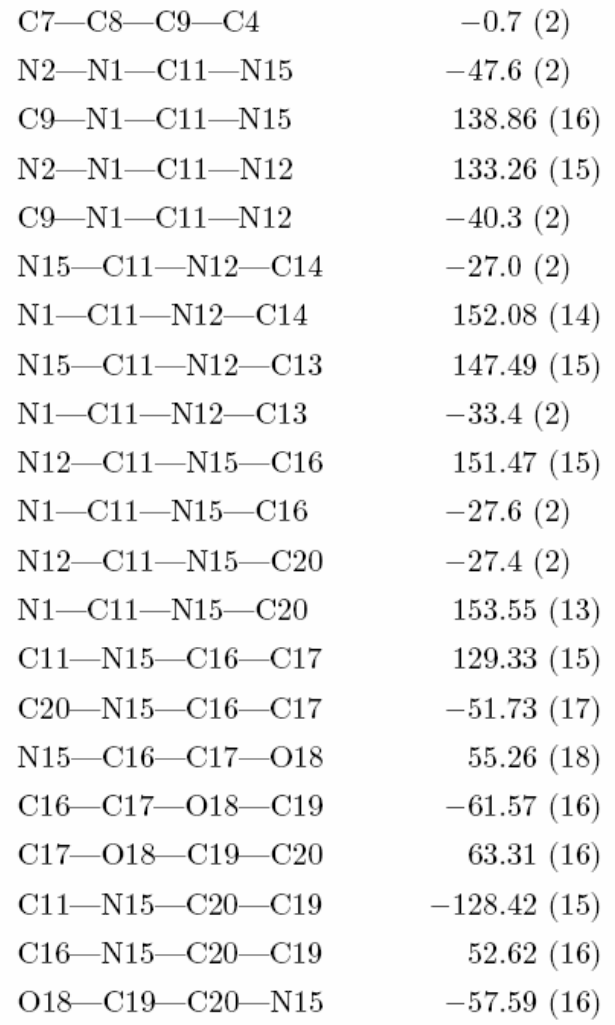

